\title{
Functional Anatomical Studies of Explicit and Implicit Memory Retrieval Tasks
}

\author{
Randy L. Buckner, ${ }^{1,3}$ Steven E. Petersen, ${ }^{1,2,3}$ Jeffrey G. Ojemann, ${ }^{1}$ Francis M. Miezin, ${ }^{1,2}$ Larry R. Squire, ${ }^{5}$ and \\ Marcus E. Raichle ${ }^{1,2,4}$ \\ 'Department of Neurology and Neurological Surgery, Washington University School of Medicine, ${ }^{2}$ The McDonnell Center \\ for the Study of Higher Brain Function, ${ }^{3}$ Department of Psychology, Washington University, ${ }^{4}$ Department of Radiology, \\ Division of Radiation Science, Mallinckrodt Institute of Radiology, Washington University School of Medicine, St. Louis, \\ Missouri 63110, and ${ }^{5}$ Veterans Affairs Medical Center, San Diego, California 92161 and Departments of Psychiatry and \\ Neurosciences, University of California at San Diego, La Jolla, California 92093
}

Across three experiments, PET scans were obtained while subjects performed different word-stem completion and FIXATION control tasks designed to study the functional anatomy of memory retrieval.

During each of three different word-stem completion scans, word-stem cues were visually presented in uppercase letters. The RECALL task required explicit retrieval of study words presented prior to the PET scan. The PRIMING task addressed the implicit effects of the prior study words without requiring intentional recall. The BASELINE task encouraged retrieval of information from a general knowledge store.

Across experiments, the similarity between study words and word stems was manipulated by presenting prescan study words in either uppercase letters identical to the stems, in lowercase letters, or auditorily. The PRIMING task was not studied with auditory presentation.

Many activations were consistent across experiments. The BASELINE task activated several regions in response to the reading and verbal-response demands of the task (visual, motor, and premotor cortices, cerebellum), as well as a left prefrontal region. The RECALL task additionally activated regions in anterior right prefrontal cortex. Bilateral occipitotemporal regions showed blood flow reductions during the PRIMING task as compared to the BASELINE task.

Activation in the right hippocampal/parahippocampal region was observed only in one experiment, and no experiment showed activation in the left medial temporal lobe.

These experiments suggest that areas of frontal cortex play a role in explicit recall and that an effect of priming may be to require less activation of perceptual regions for the processing of recently presented information.

\footnotetext{
Received Nov. 22, 1993; revised May 16, 1994; accepted June 16, 1994.

We thank Len Lich, John Hood, Tom Videen, and the staff of the Cyclotron Unit for technical assistance, Avi Snyder for clinical assistance, and Paula Jost for help with the preparation of the manuscript. Endel Tulving, Chad Marsolek, and Jeff Keating provided valuable suggestions on an earlier version of the manuscript. R.L.B. thanks Judy Ferris, an excellent teacher. This work was supported by NIH Grants NS06833, EY08775, HL13851, and AG08377, the Charles A. Dana Foundation, and the McDonnell Center for the Study of Higher Brain Function. L.R.S. was supported by the Medical Research Service of the Department of Veterans Affairs, NIMH Grant 24600, the Office of Naval Research, and the McKnight Foundation.

Correspondence should be addressed to Randy L. Buckner, Washington University School of Medicine, Campus Box 8111, St. Louis, MO 63110.

Copyright (C) 1995 Society for Neuroscience $0270-6474 / 95 / 150012-18 \$ 05.00 / 0$
}

[Key words: PET, neuroimaging, memory, explicit memory, implicit memory, frontal cortex, hippocampus, declarative memory, episodic memory, priming]

Studies of normal subjects, amnesic patients, and animal models of amnesia have demonstrated several dissociable forms of memory (Tulving, 1985; Weiskrantz, 1990; Squire, 1992; Schacter and Tulving, 1994). These studies have led to many different taxonomies of memory. One commonly agreed upon distinction is between tasks that require subjects to recollect prior facts or events intentionally (sometimes referred to as explicit or declarative memory ${ }^{1}$, and tasks in which information is accessed unintentionally (often referred to as implicit or nondeclarative memory).

Many further subdivisions of these two basic calegories have been made by different researchers. For example, some researchers distinguish between the recollections of particular events in a subject's life (sometimes referred to as episodic memory) and that subject's general knowledge of the world (sometimes referred to as semantic memory).

Implicit (or nondeclarative) memory tasks also encompass a large and heterogeneous group including various types of skill learning, classical conditioning, and priming (Tulving and Schacter, 1990; Schacter et al., 1993; Squire et al., 1993). Priming is a facilitation or bias in responding to a stimulus based on past exposure.

Brain areas putatively involved in these various forms of memory have been identified using lesion-deficit correlations in humans (Scoville and Milner, 1957; Zola-Morgan et al., 1986) and animals (Mishkin, 1982; Zola-Morgan and Squire, 1993). Perhaps the most striking anatomical distinction has been shown through the study of patients with amnesia (Scoville and Milner, 1957; Zola-Morgan et al., 1986; Victor and Agamanolis, 1990)

\footnotetext{
${ }^{1}$ Explicit memory tasks are ones that require "intentional or conscious recollection of previous experiences" (Schacter et al., 1993, p 159). Implicit memory tasks are those that demonstrate learning via some behavioral measure (i.e., faster reaction times, better correct performance) but do not require subjects to "recollect the study episode" (p 160) or to recall any facts. The distinction between declarative and nondeclarative memory has been illuminated especially by biologica dissociations and by observations of what kinds of learning and memory depend on the integrity of the medial temporal lobe and related structures. Here we use the two sets of terms interchangeably as most explicit memory tasks appear to require the medial temporal lobe memory system (Squire, 1992), but the term "explicit memory" does not itself refer to any biological systems or mechanisms (Schacter, 1987).
} 
Table 1. Stimulus format and scan tasks in the three experiments

\begin{tabular}{clll} 
& Experiment & \\
\cline { 2 - 3 } & Case-Same & Case-Change & Auditory \\
\hline $\begin{array}{c}\text { Before scan } \\
\text { Study words }\end{array}$ & see GARNISH & see garnish & hear "Garnish" \\
$\begin{array}{c}\text { During scan } \\
\text { Stem cues }\end{array}$ & see GAR & see GAR & see GAR \\
Scan 1 & FIXATION & FIXATION & FIXATION \\
Scan 2 & NO RESPONSE & NO RESPONSE & BASELINE \\
Scan 3 & BASELINE & BASELINE & RECALL \\
Scan 4 & PRIMING & PRIMING & BASELINE \\
Scan 5 & RECALL & RECALL & RECALL \\
Scan 6 & FIXATION & FIXATION & FIXATION \\
\hline
\end{tabular}

and studies of laboratory animals (Zola-Morgan and Squire, 1993) suggesting that the hippocampal formation, together with perirhinal and parahippocampal cortex, participates in explicit memory function. Amnesic patients have great difficulty in storing new information so that it can be explicitly recalled, and can also have difficulty recalling information from their recent (and in some cases distant) past. Amnesic patients, on the other hand, exhibit normal levels of performance across implicit memory tasks including priming and skill learning. These results suggest that there is some anatomical distinction between brain areas involved in the different forms of memory (Squire, 1992).

The development of modern functional imaging techniques such as positron emission tomography (PET) (Raichle, 1987) and functional magnetic resonance imaging ( $\mathrm{MRI}$ ) (Kwong et al., 1992; Ogawa et al., 1992), now make possible an extension of earlier research on memory by allowing the study of the functional anatomy of the normal human brain. These imaging techniques can measure small changes in local hemodynamics and metabolism thought to occur as the result of changes in neural activity. Accordingly, functional neuroimaging can aid in identifying areas of the brain involved in memory.

In this article, we present an analysis of data from three PET experiments that measured blood flow in human subjects performing memory retrieval tasks. One of the experiments included in this study has been previously reported (Squire et al., 1992). In all three experiments, subjects were presented study words before each PET scan, and then performed several different word-stem (word beginnings) completion tasks. These word-stem completion tasks included an explicit (or declarative) memory RECALL task (see note 1), a BASELINE task that encouraged retrieval of general knowledge of English words, and, in two of the experiments, an implicit memory retrieval task known to produce PRIMING.

In the Squire et al. (1992) report, activations of right hippocampal/parahippocampal and right frontal regions during the RECALL task were described. The rightward lateralization of these responses was surprising given the apparently verbal nature of the tasks and the pervasive left lateralization of many language-related tasks. It was speculated in this earlier report (Squire et al., 1992) that the rightward lateralization of the responses was due to the visual physical match between the beginnings of the study words and the test word stems presented during the scans themselves. This idea was supported by an independent behavioral study that used the same paradigm studied with PET (Marsolek et al., 1992, in press).
As a result of this first study (Squire et al., 1992), two further sets of experiments have been performed. In these experiments, the format of the study words was varied. This manipulation was done to address two related issues: (1) to determine whether the results from the first set of experiments generalize across different study conditions, and (2) to address specifically the speculation that the right lateralization of the observed responses was due to the visual similarity between the study words and word stems. One manipulation was to vary the visual format of the study words so that there was not a visual identity between the test word stems and the beginnings of the prescan study words; the other manipulation was to change the modality of input of the study words (words were presented auditorily).

The converging nature of the designs of these experiments

Case-Change Experiment: Voice Onset Latencies

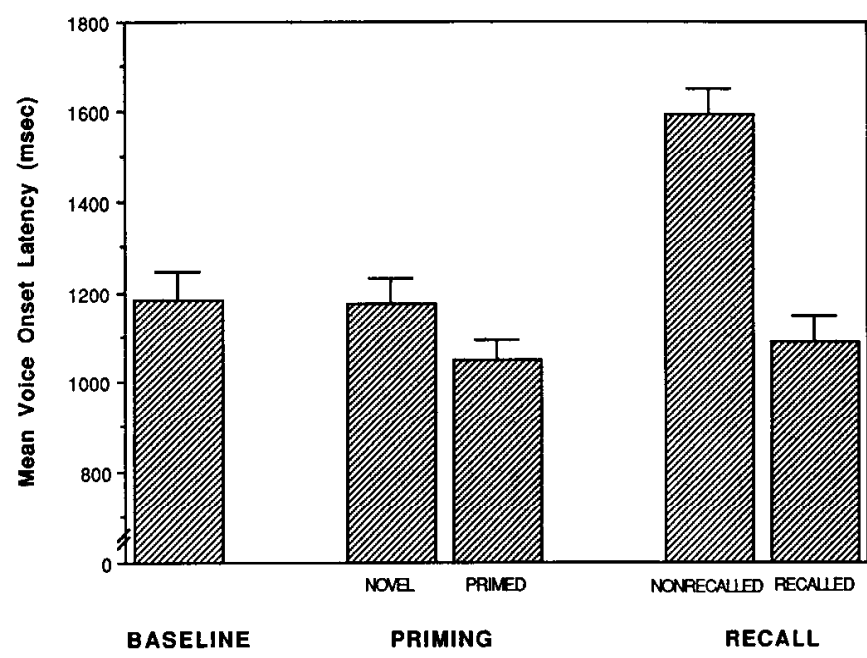

Figure 1. Mean voice onset latencies from the Case-Change experiment for the three tasks in which subjects produced words. For the PRIMING and RECALL tasks, onset latencics are further separated. For the PRIMING task, the latency to produce words that were presented during study are labeled $P R I M E D$, and the latency to produce words that were not presented during study are labeled NOVEL. For the RECALL task, the latency to produce words that were recalled from the study list are labeled RECALLED and those that were produced that were not study words are labeled NONRECALLED. Error bars show SEM. 
Table 2. RECALL - BASELINE: hypothesis generation and testing of regions defined around peaks of blood-flow increase

\begin{tabular}{|c|c|c|c|c|c|c|c|c|c|c|c|}
\hline \multicolumn{4}{|c|}{$\begin{array}{l}\text { Hypothesis-generating } \\
\text { data set } \\
(n=17)\end{array}$} & $\begin{array}{l}\text { Hypothesis- } \\
\text { testing } \\
\text { data set } \\
(n=19) \\
\text { Significance }\end{array}$ & \multicolumn{7}{|c|}{ Best estimates of locations using the combined data set $(N=36)$} \\
\hline 58 & -35 & 59 & 10 & $p<0.0001^{*}$ & -35 & 61 & 6 & 32 & 50 & 6 & R. ant. prefrontal (BA10) \\
\hline 37 & -50 & 32 & 33 & $p<0.05$ & -49 & 31 & 36 & 44 & 18 & 39 & R. prefrontal (BA 8/9) \\
\hline 36 & 3 & -43 & 36 & $p<0.01^{*}$ & 3 & -45 & 40 & -3 & -63 & 43 & Med. parietal \\
\hline 31 & -13 & 43 & 38 & $p<0.001^{*}$ & -15 & 41 & 40 & 14 & 29 & 43 & R. prefrontal (BA 8) \\
\hline
\end{tabular}

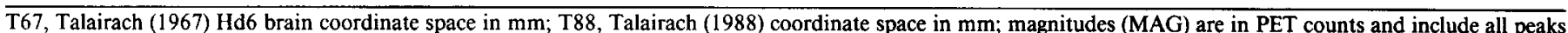

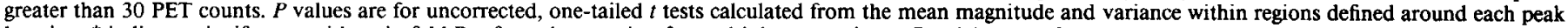
location; * indicates significance with a six-fold Bonferroni correction for multiple comparisons. R., right; L., left; Med., medial; BA, Brodmann's area.

and the specific analyses performed on this relatively large data set have allowed us to describe clear commonalities across the experiments as well as a smaller number of differences due to the study manipulations.

\section{Materials and Methods}

Three independent experiments were conducted differing mainly in regard to the format of the study word lists that were presented prior to each scan. The experiments will be referred to as the Case-Same experiment, the Case-Change experiment, and the Auditory experiment. This nomenclature expresses the relationship between the study word lists presented prior to each scan within an experiment and the word stems presented during each scan. The details of this relationship are explained below. Data from the Case-Same experiment have been rcported previously (Squire et al., 1992). Except where mentioned, the materials and methods were the same across the three experiments.

Subjects. Normal human volunteers were recruited from the local population of undergraduate and graduate students, residents, fellows, and staff at the Washington University Undergraduate Campus and Medical School and paid for participation. All subjects were (1) strongly right handed as measured by the Edinburgh handedness inventory (Raczkowski et al., 1974), (2) between the ages of 18 and 35, (3) normal or corrected-to-normal in visual acuity, and (4) had no significant neurological history. Informed consent was obtained prior to participation following guidelines approved by the Human Studies Committee (IRB) and the Radioactive Drug Research Committee of Washington University. Altogether, 40 subjects participated in one or another of the three independent experiments: Case-Same $(7$ males and 11 females, mean age $=21.9$ years), Case-Change ( 7 males and 7 females, mean age $=21.5$ years), and Auditory ( 4 males and 4 females, mean age $=23.0$ years).

Apparatus. All emission and transmission measurements were obtained using the PETT VI tomograph (Ter-Pogossian et al., 1982). PETT VI gathers seven transverse slices of data with $14.4 \mathrm{~mm}$ between the centers of adjacent slices. During PET scans, earplugs were inserted to dampen background noise, and a molded plastic facial mask was fitted to each subject's head to reduce movement (Fox et al., 1985). For the Case-Same experiment, stimuli were presented with a graphics display system (Ramtek, 9400). For both the Case-Change and Auditory experiments, stimuli were presented on a 14 inch AppleColor Hi-Res RGB monitor using a Macintosh II computer system. A microphone (Realistic, 33-2001) was taped to each subject's chest to record vocalizations. For the Case-Change experiment a second miniature microphone (Shure, A95UF) obtained voice onset latencies using a voice-activated relay (Gebrands, G134IT) fed into a computer interface (GW Instruments,
MacADIOS II). For the Auditory experiment, miniature earplug speakers were used for auditory input (Lauter et al., 1985).

A black cloth was placed around the monitors to reduce extraneous visual input. The experimental room lights were dimmed during scans. Cooling fans provided low-level background noise.

PET scanning techniques. The PET scanning activation methodology developed at Washington University was used (Fox et al., 1988; Mintun et al., 1989; Petersen et al., 1989).

Subjects were positioned within the tomograph so that they could comfortably view a computer monitor placed 12-18 inches from their eyes. A lateral skull $x$-ray was taken to verify head alignment and to identify the glabella and inion as markers to locate the position of the transverse plane intersecting the anterior and posterior commissures (Talairach et al., 1967; Fox et al., 1985). Using stereotactic techniques (Fox et al., 1985), the approximate location of the hippocampus was identified. The position of each subject in the scanner was adjusted so that the slice nearest to the hippocampus would sample as closely as possible activity in the hippocampus.

${ }^{15} \mathrm{O}$-labeled water (half-life of $123 \mathrm{sec}$ ) was used as a blood-flow tracer and administered as an intravenous bolus injection. Six scans were performed sequentially on each subject, with each scan spaced about 10 min apart to allow nearly complete decay of the ${ }^{15} \mathrm{O}$ between scans. Each of the six scans was acquired during $40 \mathrm{sec}$ while the subjects performed one of several tasks (see behavioral procedures for task descriptions). The PETT VI system was used in the low-resolution mode, and images were reconstructed to $18 \mathrm{~mm}$ full width at half-maximum using filtered back-projection (Yamamoto et al., 1982). As blood-flow increases are known to be a linear function of radiation counts for scans of less than 1 min duration, measurements of arterial blood radioactivity following ${ }^{15} \mathrm{O}$ watcr injection were not made (Herscovitch et al., 1983; Fox et al., 1984; Fox and Mintun, 1989). Rather, local radiation counts were used as an estimate of local blood flow. For simplicity, the remainder of the text refers to changes in tissue radioactivity as changes in blood flow and quantifies the radiation in terms of PET counts.

All PET images were normalized by linear scaling for global blood flow to the set value of 1000 PET counts so that fluctuations in global blood flow would not obscure local changes induced by task manipulations (Fox et al., 1987). Each image was transformed into a standardized stereotactic space based on the Hd6 brain of the Talairach 1967 atlas (Talairach et al., 1967) and then also rescaled into the stereotactic space from the more recently available atlas (Talairach and Tournoux, 1988). Voxels in the transformed images measured $2.0 \times 2.0 \times 2.0$ $\mathrm{mm}$. Within the text, all coordinates are reported $(x, y, z)$ in both the coordinate space of the Hd6 brain of the Talairach 1967 atlas (T67) and of the Talairach 1988 atlas (T88).

To isolate local changes in blood flow, subtraction images were obtained by subtracting blood-flow data from one scan (CONTROL) from

Figure 2. Horizontal blood-flow sections from the RECALL - BASELINE and RECALL - FIXATION summed subtraction images are shown in Talairach (1988) space with the z-coordinate labels. For each subtraction pair, the top seven sections show blood-flow increases and the bottom seven sections show blood-flow decreases. These sections show all of the blood-flow changes that differed between the two scan tasks. 


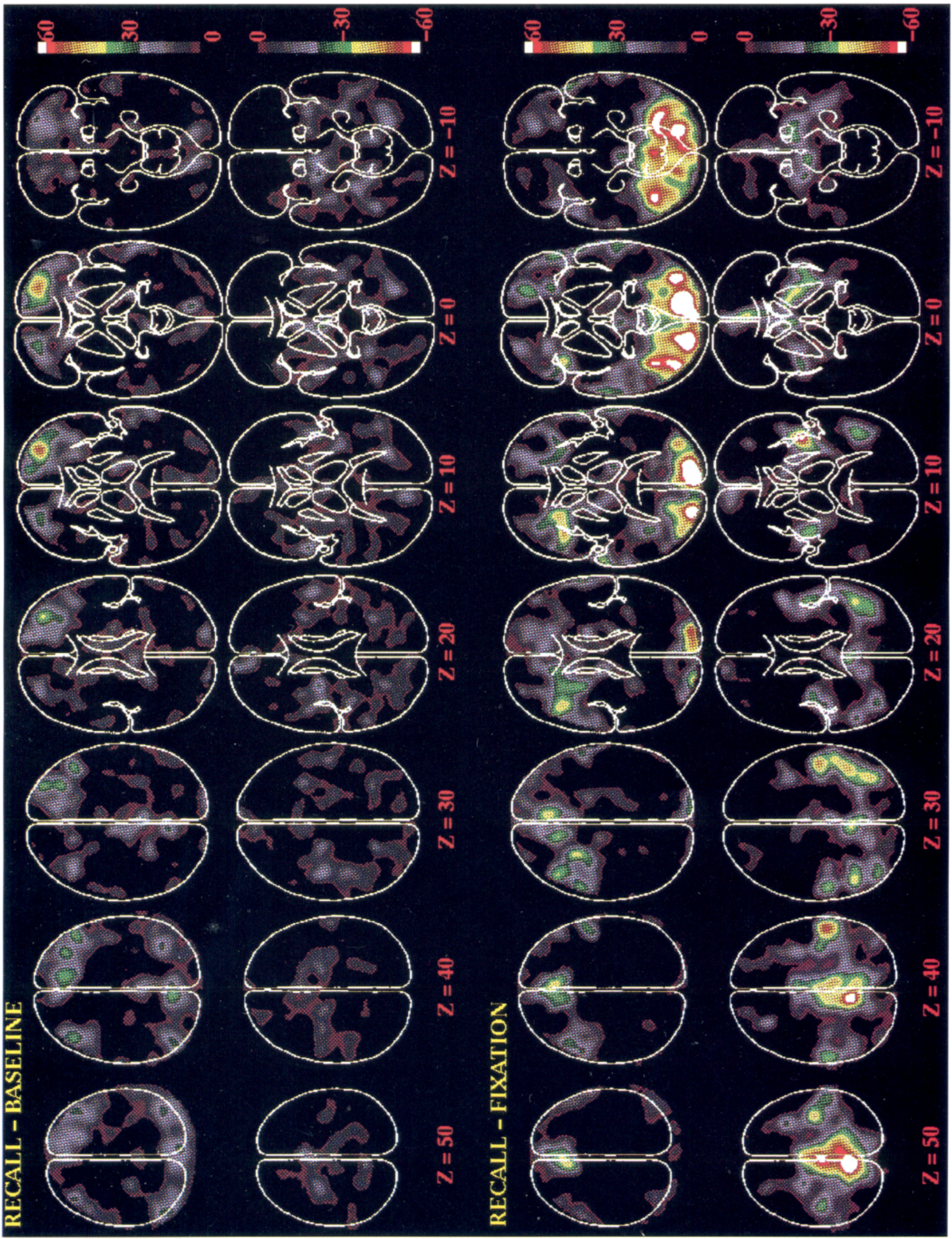


a second scan (ACTIVE). All subtraction pairs were screened for subject movement before the data were examined for task-induced blood-flow changes. Scan pairs with obvious movement artifact were eliminated from further analysis. Within the text, reported sample sizes refer to the total number of usable subtraction pairs, not the number of pairs collected or the number of subjects tested. These numbers often differ, as many subtraction pairs were eliminated because of movement. Because the scans were done while different tasks were being performed, the ACTIVE

CONTROL subtraction images reflect blood-flow changes induced by the processing demands that differed between the ACTIVE and CONTROL tasks. Signal-to-noise properties of the subtraction images were increased by averaging data from several subjects (Fox et al., 1984; Mintun et al., 1989). Subtraction images from individual subjects were also created and used to assess between-subject variability in the magnitude of activations in specific regions.

Behavioral procedures. The goal of the behavioral tasks was to isolate processing demands related to explicit and implicit memory retrieval. To accomplish this goal within the constraints of PET, different versions of the word-stem completion task (Graf et al., 1984) were used: two memory retrieval tasks (RECALL and PRIMING) and a set of control tasks (BASELINE and NO RESPONSE). The control tasks were designed to incorporate subsets of the processing demands required by the memory retrieval tasks. A third low-level control task (FIXATION) was also used for additional comparisons.

With one exception, the general format for all of the behavioral tasks was the same. Beginning 3-4 min prior to a scan, subjects were presented a list of 15 study words ( $3.5 \mathrm{sec}$ per word). Then, about $15 \mathrm{sec}$ prior to the scan, one of the behavioral tasks was begun, which continued throughout the duration of the $40 \mathrm{sec}$ PET scan. The exception to this format occurred in the Auditory experiment where study words were not presented prior to the FIXATION task scan.

The study words were presented twice in a different random order, always with three filler words presented at the beginning of the list and two filler words presented at the end of the list to reduce primacy and recency effects. Thus, each study list of 15 words contained 10 target words. Study words were between four and eight letters in length, of moderate frequency (50 per million; Kucera and Francis, 1967), and each had a three-letter word beginning that was different from the first three letters of any other study word. Subjects were asked to rate each word, on a scale from 1 to 5 , according to how much they liked the word meanings. This manipulation insured that each word was attended. No indication was given that the study list was part of a memory task.

During the scans, one of the five tasks was administered. For the RECALL, PRIMING, BASELINE, and NO RESPONSE tasks, subjects were visually presented 20 three-letter uppercase word stems (e.g., GAR) ( $3.0 \mathrm{sec}$ per stem with a $0.5 \mathrm{sec}$ interval between stems). The tasks differed from one another in two respects: (1) the relationship between the word stems and the study words and (2) the instructions that the subjects received telling them what to do with the word stems. For the FIXATION control task, subjects were instructed to look at a centrally presented cross hair for the duration of the PET scan and no word stems were presented.

In both the RECALL and PRIMING memory tasks, 10 of the 20 word stems could be completed to form study words. Thus, if subjects were presented the study word "GARNISH" before the scan, the stem "GAR" was presented during the scan. The only difference between the RECALL and PRIMING tasks was the instructions. For the RECALL task, subjects were instructed to try to recall study list words that began with the same three letters as the word stems and to guess if they were not sure. The RECALL task demanded that the subjects intentionally and consciously retrieve words from memory. Amnesic patients are impaired at this task (Graf et al., 1984). For the PRIMING task, subjects were instructed to complete the word stems with the first word that came to mind, and no mention was made that the stems could be completed to form study words. Under these conditions, it is known that normal subjects and amnesic patients have a similar tendency to produce words from the earlier study list (Warrington and Weiskrantz, 1974; Graf et al., 1984). The PRIMING task did not require that the subjects be aware that they were retrieving study words.

In both the BASELINE and NO RESPONSE control tasks, none of the word stems could be completed to form study words. That is, if subjects had studied the word "GARNISH" before the scan, "GAR" was not presented during the scan. For the BASELINE control task, the instructions were identical to the PRIMING task: subjects were instructed to complete the word stems with the first word that came to mind. Unlike the PRIMING or RECALL tasks, however, subjects could not form study words from the stems. In this respect, the BASELINE task did not demand or encourage the subjects to retrieve any recently studied words. The BASELINE task required the subjects to process the stems visually, generate and access appropriate words that completed the stems, and pronounce those words. The requirement to access appropriate words is a form of semantic memory retrieval, as the subjects must retrieve words they had learned during the course of their lifetime. Processing demands related to semantic memory retrieval were also required by the RECALL and PRIMING tasks. Blood-flow measurements obtained during the BASELINE task could be subtracted away from blood-flow measurements obtained during the RECALL and PRIMING tasks to isolate local changes in blood flow caused by task demands related to explicit and implicit memory retrieval. For the NO RESPONSE control task, subjects were instructed to simply look at the word stems and do nothing. Blood flow measurements obtained during the NO RESPONSE task could be subtracted away from measurements made during the BASELINE task to isolate local blood-flow changes caused by the speech production demands of the BASELINE task.

Across the three experiments, the prescan presentation format of the study words was varied (Table 1). In the Case-Same experiment, study words were presented visually in uppercase letters (e.g., GARNISH) $\left(\approx 0.08^{\circ}\right.$ wide per letter); in the Case-Change experiment, the study words were presented visually in lowercase letters (e.g., garnish) $\left(\approx 0.08^{\circ}\right.$ wide per letter); and in the Auditory experiment, the study words were auditorily presented in a male voice (e.g., "garnish").

During the scans for all three experiments, the word stems were visually presented in uppercase letters (e.g., GAR) (Table 1). By presenting visually identical word stems across the three experiments, but varying the format of the study words, three slightly different retrieval situations were created. In the Case-Same experiment, the word stems and the beginnings of the study words were perceptually identical. In the CaseChange experiment, both study words and word stems were presented visually but, because the study words were presented in lowercase and the word stems in uppercase, they were not perceptually identical. In the auditory experiment, the study words were presented aurally, and the word stems were presented visually.

About $1 \mathrm{hr}$ before the beginning of the first scan of each experiment, subjects were acquainted with the BASELINE task by completing about five stems to form words. Study word presentation began about $3-4$ min prior to each scan, as described above. Immediately after the presentation of study words, and about $1 \mathrm{~min}$ before each scan, instructions for the appropriate task were given.

The order of the six PET scans was the same for most subjects in the Case-Same and Case-Change experiments (Table 1). The first and sixth scans used the FIXATION task and the second, third, fourth, and fifth scans were the NO RESPONSE, BASELINE, PRIMING, and RECALL tasks, respectively. However, for the first eight subjects in the CaseSame study, the NO RESPONSE, BASELINE, PRIMING, and RECALL tasks were administered in a random order. Finally, the Auditory experiment omitted the NO RESPONSE and PRIMING tasks in order to repeat the two tasks specifically related to explicit memory retrieval. Thus, the order of the tasks across the six scans in the Auditory experiment was FIXATION, BASELINE, RECALL, BASELINE, RECALL, FIXATION (Table 1).

\section{Results}

\section{Behavioral results}

In the RECALL task, subjects correctly recalled $76.4 \%, 73.0 \%$, and $61.7 \%$ of the words in the Case-Same, Case-Change, and Auditory experiments, respectively. An ANOVA revealed a significant main effect of recall performance across experiments $(F[2,33]=5.02, p<0.05)$. Post hoc $t$ tests found that performance in the Auditory study was significantly poorer than performance in either of the other two studies $(t[20]=2.33$ and $t[24]=3.03$, all $p<0.05)$.

In the PRIMING task, subjects completed $71.5 \%$ and $51.5 \%$ of the word stems to form study words in the Case-Same and Case-Change experiments, respectively. An ANOVA revealed a significant main effect of experiment $(F[1,24]-9.26, p<$ $0.01)$. For the two experiments combined, primed words were 
produced much more frequently than would be expected by chance $(7.5 \%$ as demonstrated by Squire et al., 1992, for this word list $)(t[25]=14.26, p<0.0001)$.

Voice onset latencies were recorded for the Case-Change experiment (Fig. 1). The mean latency ${ }^{2}$ to produce words during the BASELINE task was $1181 \mathrm{msec}$. Latencies for words produced in the PRIMING task were divided into two sets: the first set consisted of those words that were identical to words presented in the study list (PRIMED), and the second set consisted of the novel words that were produced in response to word stems that could not be completed to form words from the study list (NOVEL). Instances in which a novel word was produced when a PRIMED word could have been produced were not included in either set. The mean latency for NOVEL words was $1176 \mathrm{msec}$, nearly identical to latency for words in the BASELINE task. The mean latency for PRIMED words was $1045 \mathrm{msec}$, significantly less than the latency for NOVEL words (paired $i[13]=3.18, p<0.01$ )

Likewise, the words produced in the RECALL task were divided into two sets: the first set included correctly recalled words (RECALLED), and the second set included novel words absent from the study list (NONRECALLED). NONRECALLED words occurred when a word stem could not be completed to form a study word, but the subjects produced a word anyway. Instances in which a novel word was produced when a study word could be RECALLED were not included in either set. The mean latency for RECALLED words was $1085 \mathrm{msec}$, and the mean latency for NONRECALLED words was significantly slower at $1592 \mathrm{msec}$ (paired $t[13]=7.42, p<0.0001$ ).

\section{PET blood-flow results}

Rationale for analysis

Analyses werc conducted primarily using three subtraction pairs: RECALL - BASELINE, PRIMING - BASELINE, and BASELINE - FIXATION. The RECALL - BASELINE subtraction isolated blood-flow changes related to the explicit memory retrieval demands required by the RECALL task. The PRIMING - BASELINE subtraction isolated blood-flow changes related to the priming, or implicit memory retrieval, demands of the PRIMING task. The BASELINE - FIXATION subtraction isolated blood-flow changes produced by the general demands of the word-stem completion task. The NO RESPONSE task was not analyzed for reasons described below.

Analyses of the three primary subtraction pairs were carried out by identifying blood-flow changes common to all three experiments (Case-Same, Case-Change, and Auditory). This was accomplished by combining the data from the three experiments into single large- $N$ images. Additionally, data from individual experiments were analyzed independently in order to localize blood-flow changes specific to single experiments. The data from the Case-Same experiment have been previously analyzed as an individual experiment (Squire et al., 1992).

In addition to the three primary subtraction pairs identified above, the RECALL - FIXATION and PRIMING - FIXATION subtraction pairs were used to validate the assumptions

\footnotetext{
For each subject, mean latencies were computed for each condition using all trials in which a voicing error did not occur. Voicing errors occurred when subjects spoke too softly for the voice relay, when the voice relay triggered to a noise other than the voice, or when the subject preceded a word with a nonword vocalization such as "um." Within the text, mean latencies refer to the grand mean for all subject means, and statistical analyses were based on subject means.
}

of the subtraction methodology. Specifically, the RECALL and PRIMING tasks were designed to include all of the demands of the BASELINE task, plus additional demands related to memory retrieval. An assumption made in using the RECALL - BASELINE and PRIMING - BASELINE subtraction pairs was that processing demands, and hence blood-flow changes, related to the speech production demands of the BASELINE task were contained within the PRIMING and RECALL tasks. To test this assumption, blood-flow changes identified within the BASELINE - FIXATION subtraction pair were looked for in the PRIMING - FIXATION and RECALL - FIXATION subtraction pairs.

Finally, in some instances, areas showing blood-flow change in one subtraction image (e.g., RECALL - BASELINE) were also examined in other subtraction images (e.g., PRIMING BASELINE and BASELINE - FIXATION) in order to track more precisely the behavior of blood flow within that area across task conditions.

\section{RECALL - BASELINE subtraction}

Regions active across experiments. Initial analyses were conducted to determine local regions of blood-flow change that were common to all three experiments in the RECALL task, using the BASELINE task as a control. Accordingly, a combined data set of RECALL - BASELINE subtraction pairs $(N=36)$ from all three experiments was divided into two sets, a "hypothesisgenerating data set" $(n-17)$ and a "hypothesis-testing data set" $(n=19)$. Using a center-of-mass search algorithm (Mintun et al., 1989), all peaks of blood-flow change greater than 30 PET counts were localized within the summed image of the hypothesis-generating data set (Table 2). Regions of interest were then defined around locations that corresponded to these peaks (six increases and three decreases). Each region included all voxels within a 7 -voxel-diameter sphere centered at each location. Finally, mean magnitudes of blood-flow change and variance were calculated for the volume defined by each region within the second, independent hypothesis-testing data set. Using a onesample $t$ test, mean change in blood flow was significantly greater than zero for the six regions of blood-flow increase (Table 2). The three regions of blood-flow decrease were not significant.

Best estimates of the locations of peak blood-flow increase within these six replicated regions were obtained using the center-of-mass search algorithm on the combined data set $(N=36)$ (Table 2, Fig. 2). A region located within anterior right prefrontal cortex, at or near Brodmann's area 10, in frontal-polar cortex, was significantly increased. Blood flow in three regions in right superior prefrontal cortex and one analogous region in left prefrontal cortex, all at or near Brodmann's area 8, were also increased. Mean blood flow in a region in medial parietal cortex also showed a significant incrcase. The absence of local regions of blood-flow decrease suggests that the processing demands added by the RECALL task, as compared to the BASELINE task, primarily caused additional brain regions to be recruited and did not eliminate or suppress regions activated by the BASELINE task. Of the six regions identified in the RECALL - BASELINE comparison, all were increased above zero in each of the three experiments, and there were no interactions across experiments. Thus, these regional blood-flow increases were unaffected by changes in study-word format.

Regions active in specific experiments. To determine regions 


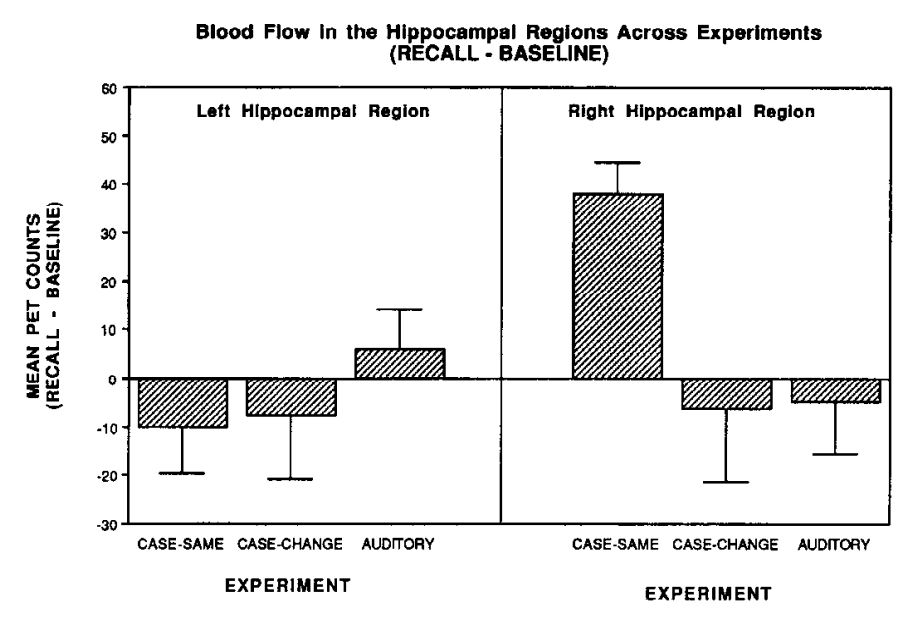

Figure 3. Mean regional blood-flow changes within the left and right hippocampal regions, including the parahippocampal gyrus, are shown for the RECALL - BASELINE subtractions. The two panels display data for the left and right hippocampal regions, respectively. Each bar represents data from one experiment, +SEM.

of blood flow change in the RECALL - BASELINE subtraction that were present only in individual experiments, three summed images were generated that included only the subtraction pairs from a single experiment (Case-Samc, $n=14$; Case-Change, $n$ $=10$; Auditory, $n=12$ ). Two peaks of blood-flow increase were identified that were not found in the combined data set. The first peak $(\mathrm{T} 67=21,35,-6 ; \mathrm{T} 88=-19,22,-6)$, located at or near Brodmann's area 47, was found in the Auditory experiment (peak magnitude of 38 PET counts) but was not found in either of the other two experiments. There were too few subtraction pairs in the Auditory data set to provide for a second, hypothesis-testing data set. Thus, it is not clear that this finding is replicable.

The second peak, found within the Case-Same experiment, was located within the area of the right hippocampus and parahippocampal gyrus (T67 $=-23,-15,-4 ; \mathrm{T} 88=21,-31,-4$ ) (peak magnitude of 43 PET counts). Previous analysis of this response that included replication in a second, hypothesis-testing data set showed that this blood-flow increase was reliable (see Squire et al., 1992). Extending this earlier analysis to include data from the Case-Change and Auditory experiments, a spherical 7-voxel region was defined around this peak and applied to all three data sets. Blood flow in this region did not iñcrease in either the Case-Change or the Auditory experiments (Fig. 3). An ANOVA revealed a significant main effect of experiment for mean blood flow in this region $(F[2,33]=6.14, p<0.01)$, and post hoc $t$ tests indicated that the regional blood flow was greater in the Case-Same experiment than in either of the other two experiments $(t[22]=2.97$ and $t[24]=3.52$, both $p<0.01)$.

A symmetric left hippocampal region was also examined in each of the three data sets (center of region T67 $=23,-15,-4$; $\mathrm{T} 88=-21,-31,-4)$. Blood flow in this left hippocampal region did not exhibit a significant change from zero in any of the three experiments (all $p>0.3$ ) (Fig. 4). A similar result was obtained when the data from the three experiments were com- bined to increase power ( $N=36, M=-4.1$ PET counts, onesample $t[35]=0.72, p=0.48$ ), suggesting that net neuronal activity did not increase in the left hippocampal region during the RECALL task.

It is possible that some aspect of the BASELINE task produced activation within the left or right hippocampal regions and thus decreased the ability to detect changes caused by the additional demands of the RECALL task. To address this possibility, an analysis of the left and right hippocampal regions was carried out using the RECALL - FIXATION subtraction pairs. No changes in blood flow were found in the hippocampal regions bilaterally for the combined RECALL - FIXATION data set $(N=32, M=-2.1$ and $M=-3.8$ PET counts, $t[31]$ $=0.33, t[31]=0.54, p>0.5$ for the right and left hippocampal regions, respectively) (Fig. 2). As would be expected from the increased blood flow observed in the right hippocampal region in the Case-Same RECALL - BASELINE subtraction, an ANOVA revealed a significant main effect of experiment for mean blood flow in this region in the RECALL - FIXATION subtraction $(F[2,29]=3.96, p<0.05)$. Blood flow increased above zero only in the Case-Same experiment.

\section{PRIMING - BASELINE subtraction}

Regions active across experiments. Using the same hypothesisgeneration and hypothesis-testing approach just described for the RECALL - BASELINE subtraction, a combined data set of PRIMING - BASELINE subtraction pairs $(N=29)$ was divided into two sets. Because the Auditory experiment did not contain the PRIMING task, this combined data set included only data from the Case-Same and Case-Change experiments. No peak of blood-flow increase greater than 30 PET counts in magnitude was identified in the hypothesis-generating data set $(n=14)$. Therefore, no further testing was done to localize regions of blood-flow increase. Six peaks of blood-flow decrease were greater than 30 PET counts in magnitude. Seven-voxel spherical regions were defined around each of these peaks. Using a one-sample $t$ test with an independent hypothesis-testing data set $(n=15)$, mean change in blood flow showed a trend toward decreasing below zero in three of the six regions $(p<0.1)$. Regions that showed a trend were in the white matter medial to the caudate nucleus $(\mathrm{T} 67=9,21,20 ; \mathrm{T} 88=-8,7,21)$, in posterior insular cortex $(\mathrm{T} 67=39,3,-8 ; \mathrm{T} 88=-35,-18$, $-9)$, and in right posterior cortex (T67 $=-31,-45,4 ;$ T $88=$ $28,-63,4)$.

The location of the right posterior blood-flow reduction was in occipitotemporal cortex close to the right posterior reduction identified previously in the Case-Same experiment (Squire et al., 1992). For this reason, and for theoretical reasons that suggest perceptual priming is based on facilitation of information processing in posterior sensory cortex (Gabrieli et al., 1990; Keane et al., 1992; Marsolek et al., 1992), further analyses were conducted. To address questions of laterality, the largest peak blood-flow decrease in left posterior cortex was also identified in the hypothesis-generating data set $(n=14)$ even though no left posterior reduction was greater than 30 PET counts in magnitude. The largest posterior left-lateralized decrease localized to occipitotemporal cortex $(\mathrm{T} 67=45,-53,-4 ; \mathrm{T} 88=-41$, 


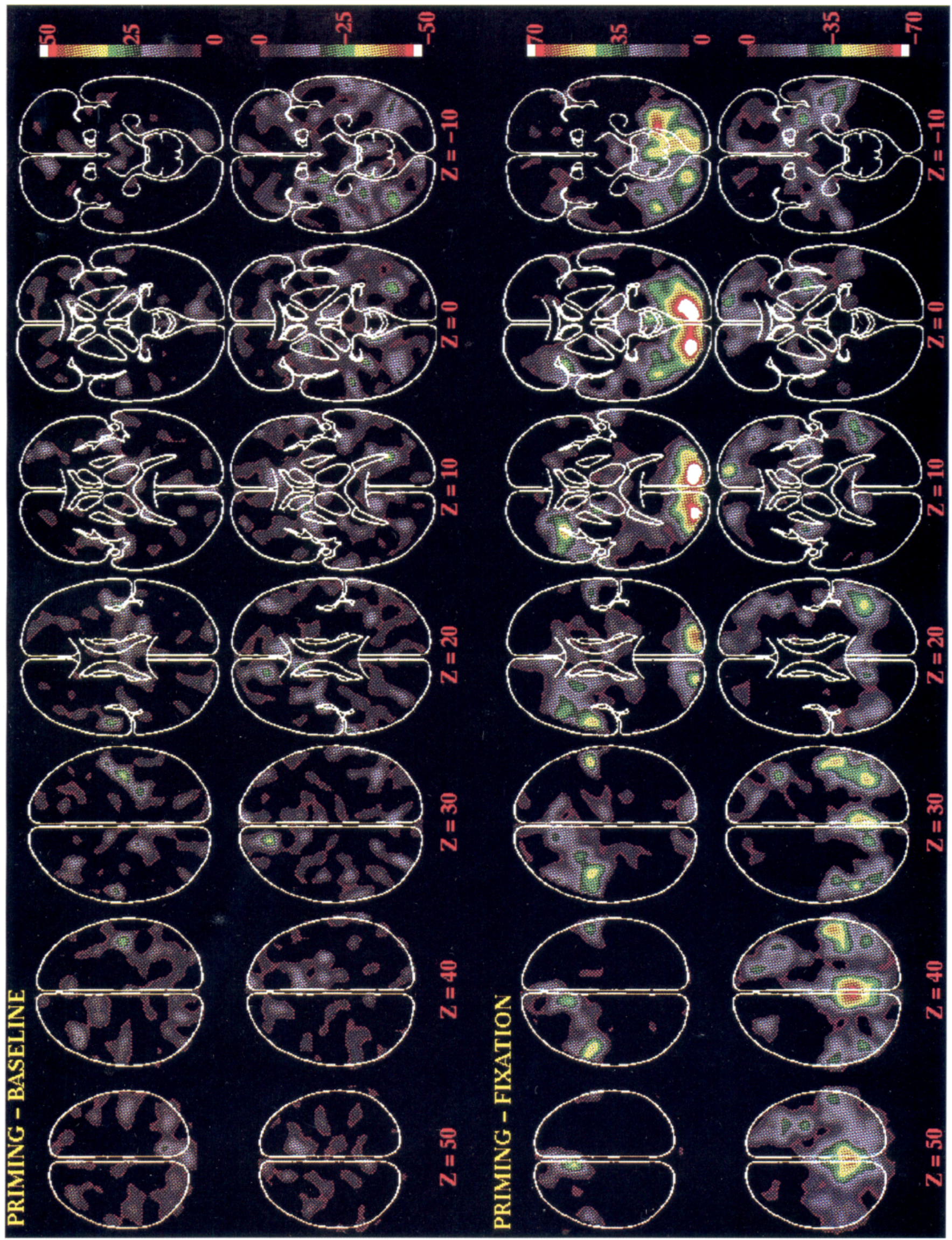




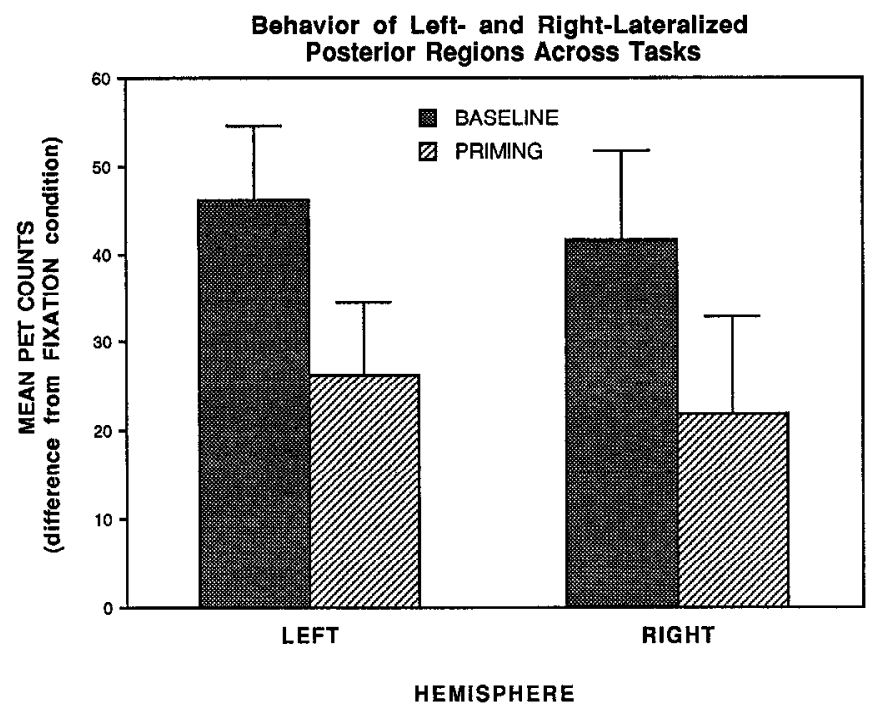

Figure 5. Mean regional blood-flow changes within bilateral posterior regions identified in the PRIMING - BASELINE subtraction (see text) are shown for the BASELINE - FIXATION and PRIMING - FIXATION subtractions. The data include subtraction pairs from the CaseSame and Case-Change experiments. Compared to the FIXATION task, blood flow is increased in both left- and right-latcralized rcgions across the BASELINE and PRIMING tasks. Blood-flow increases are not as large in the PRIMING task compared to the BASELINE task. This reduction in activation in the PRIMING task, in comparison to the BASELINE task, shows a PET correlate of priming.

$-71,-4)$. Blood flow within a 7-voxel region defined around this location was found to be significantly decreased below zero in the independent, hypothesis-testing data set (one-sample $t$ [14] $=2.22, p<0.05$ ). Using combined data from both the hypothesis-generating and hypothesis-testing data sets $(N=29)$, the right posterior reduction was localized to $T 67=-31,-45$, $4 ; \mathrm{T} 88=28,-63,4$, and the left posterior reduction identified by the post hoc analysis localized to $\mathrm{T} 67=41,-51,-8 ; \mathrm{T} 88$ $--37,-69,-9$.

Although these two reductions were not significant when a Bonferroni correction was applied and the left-lateralized reduction was analyzed post hoc, it should be noted that the two reductions were identified as the first- and third-largest peak blood-flow decreases in the large- $N$ combined image.

Using within-subject data from the BASELINE - FIXATION and PRIMING - FIXATION subtractions $(n=22)$, behavior of blood flow was tracked in the bilateral posterior regions across tasks. For both regions, blood flow was increased in both the BASELINE and the PRIMING tasks, as compared to the FIXATION task (Fig. 5). This increase was larger in the BASELINE task than in the PRIMING task, such that bloodflow decreases were observed in the PRIMING - BASELINE subtraction.

To characterize further the behavior of the hippocampal regions studied in the RECALL - BASELINE subtraction, the same right and left hippocampal regions were examined in the PRIMING - BASELINE subtraction pairs (Fig. 6). Blood flow in the left hippocampal region did not change in either of the two experiments $(t[15]=0.35, p=0.73 ; t[12]=1.17, p=0.26)$. Blood flow in the right hippocampal region increased in the Case-Same experiment but did not increase in the Case-Change experiment. An ANOVA revealed a trend toward a main effect of experiment $(F[1,27]=2.99, p<0.1)$ and post hoc $t$ tests
Blood Flow In Hippocampal Regions Across Experiments (PRIMING - BASELINE)

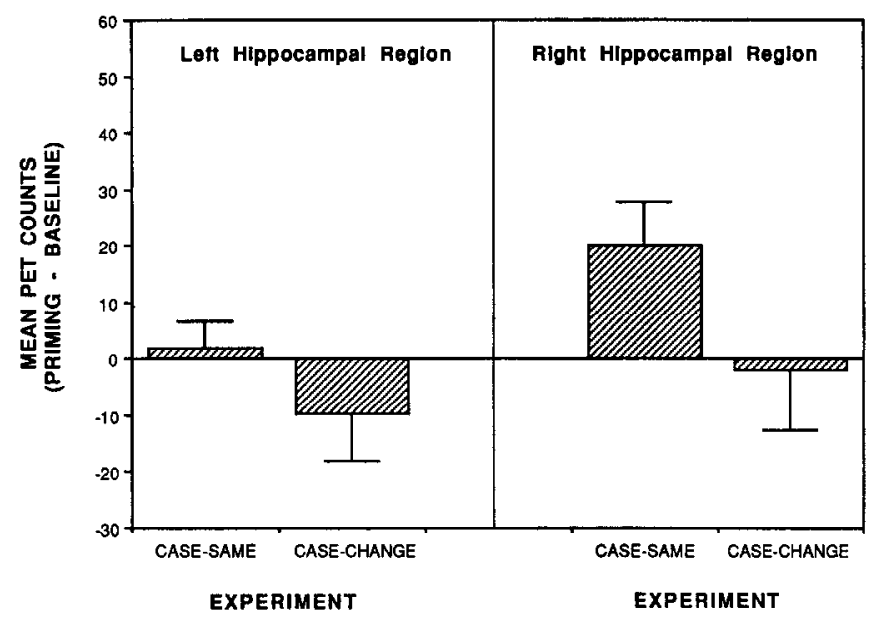

Figure 6. Mean regional blood-flow changes within the left and right hippocampal regions, including the parahippocampal gyrus, are shown for the PRIMING - BASELINE subtractions. The two panels display data for the left and right hippocampal regions, respectively. Each bar represents data from one experiment, + SEM.

revealed that regional blood flow in the Case-Same experiment was significantly greater than zero (one-sample $t[15]=2.57, p$ $<0.05$ ), while the Case-Change experiment was not (one-sample $t[12]=0.21, p=0.84)$.

Regions active in specific experiments. As an additional way of establishing that the blood-flow reductions observed in posterior cortex were not spurious, the regions obtained from the analysis of the combined data set were examined in each of the experiments separately (Case-Same, $n=16$; Case-Change, $n=$ 13). Blood flow in both regions in both experiments was significantly decreased below zero (for the Case-Same and CaseChange experiments, respectively: right region, $M=-21.4$ and $M=-23.3$ PET counts, one-sample $t[15]=3.71$ and $t[12]=$ 2.35; left region, $M=-26.8$ and $M=-21.2$ PET counts, onesample $t[15]=2.61$ and $t[12]=2.25$; all $p<0.05$ ). These data suggest that the reductions identified in the combined data set were reliable across experiments. Furthermore, comparisons of the magnitudes of blood-flow change within these posterior regions did not show a significant difference between the two experiments. $^{3}$

Independent and separate analysis of the posterior regions in the summed images from the Case-Same $(n=16)$ and CaseChange $(n=13)$ experiments revealed that the right-lateralized peak decrease in blood flow localized to two slightly different regions in the two experiments. This difference was ignored in the previous analysis. The Case-Same experiment localized this decrease to $\mathrm{T} 67=-33,-47,-4 ; \mathrm{T} 88=30,-65,-4$, and the Case-Change experiment localized this decrease to $\mathrm{T} 67=-29$, $-43,6 ; \mathrm{T} 88=26,-60,6$. The left-lateralized blood-flow decreasc localized to ncarly the same coordinates in both experiments (T67 $=41,-49,-6$; T $88=-37,-67,-6$ in the CaseSame experiment and $\mathrm{T} 67=43,-51,-10 ; \mathrm{T} 88=-39,-69$, -11 in the Case-Change experiment). In the combined data set,

\footnotetext{
${ }^{3}$ Squire et al. (1992) originally reported the presence of the right-lateralized reduction. The right-lateralized reduction was chosen for analysis because the magnitude of the reduction was the largest found in the data set. The left-lateralized reduction was present in that data set but, being smaller in magnitude, it ranked as the fourth-largest reduction and was not described in the earlier report.
} 
Table 3. BASELINE - FIXATION: hypothesis generation and testing of regions defined around peaks of blood-flow increase

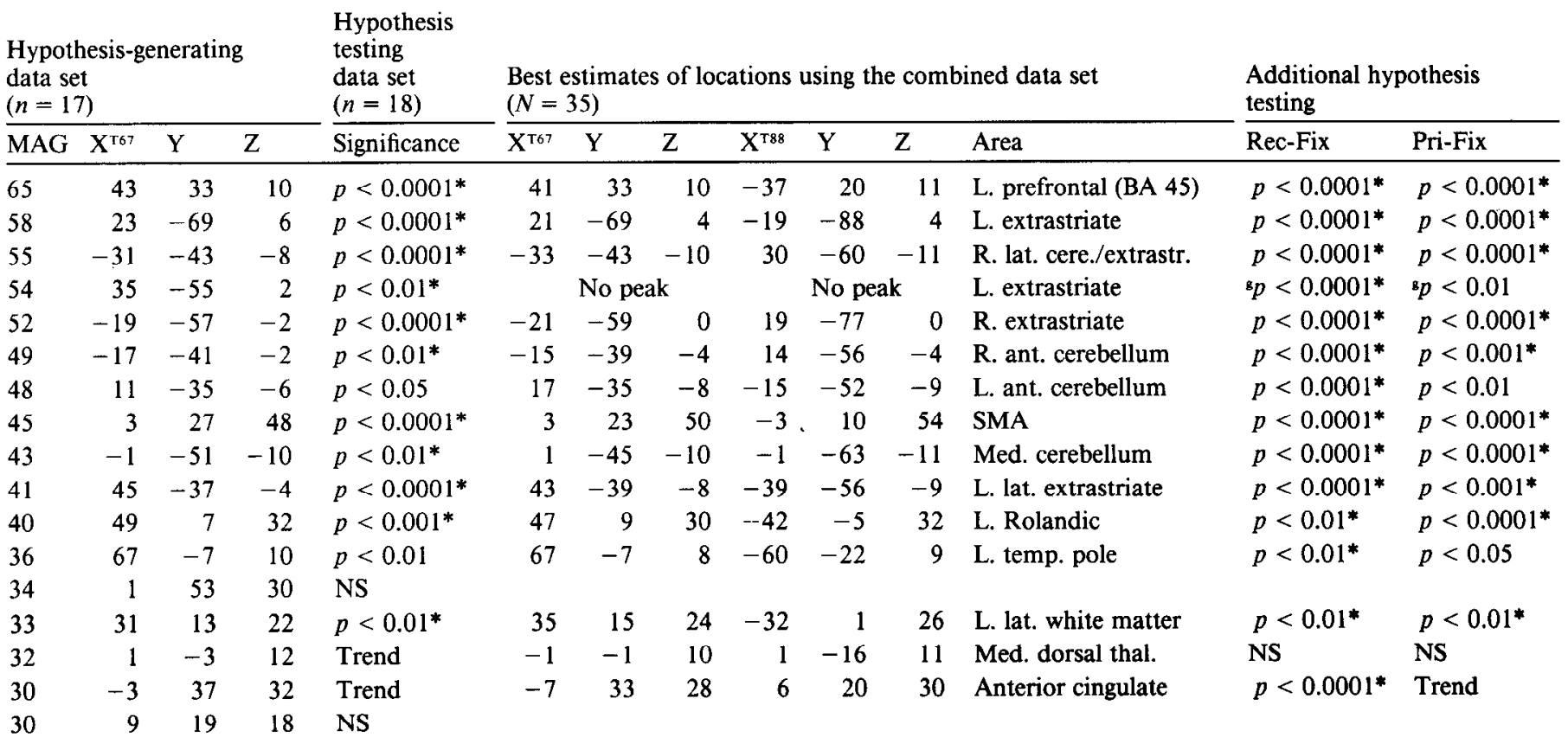

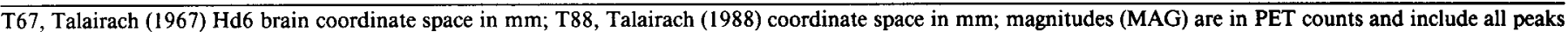

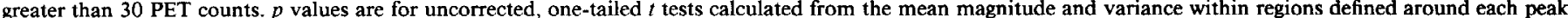

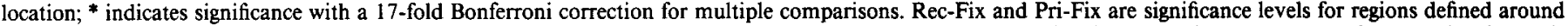

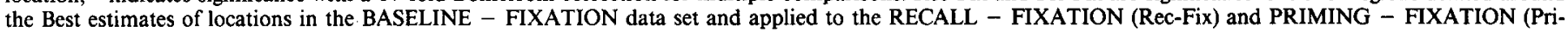
Fix) data sets. $g$, coordinates taken from the hypothesis-generating data set; R., right; L., left; Med., medial; BA, Brodmann's area.

which ignored the between-experiment variability, the rightlateralized decrease was attenuated, making it seem approximately equal in magnitude to the left-lateralized decrease. However, when the Case-Same and Case-Change experiments were inspected individually, the peak magnitudes of the right decreases were larger than the left decreases ( -38 PET counts vs -29 PET counts for the Case-Same experiment and -30 PET counts vs -21 PET counts for the Case-Change experiment). These results are consistent with the earlier observation of a right-sided blood-flow decrease in the PRIMING - BASELINE subtraction in the Case-Same experiment (Squire et al., 1992).

\section{BASELINE - NO RESPONSE and NO RESPONSE - FIXATION subtractions}

The NO RESPONSE task was designed to have subjects view visual word stems but not engage in covert or overt word production. However, in response to debriefing questions, after the scans, almost all of the subjects indicated that they tried to make the stems into words-even though they were not explicitly instructed to do so. In other words, for the NO RESPONSE task, subjects apparently performed the BASELINE task subvocally. This conclusion is supported by the finding that the blood-flow changes in the NO RESPONSE - FIXATION subtraction were qualitatively similar to those observed in the BASELINE - FIXATION subtraction (see next section), with the exception that in the NO RESPONSE - FIXATION subtraction no blood-flow increases were observed in the cerebellum or right-lateralized Rolandic cortex (Fig. 7). This being the case, the NO RESPONSE task was not analyzed further, and the FIXATION task was used as a control for the BASELINE task.

\section{BASELINE - FIXATION subtraction}

Regions active across experiments. Following the same procedure as in the RECALL - BASELINE subtraction, 35 image pairs from the BASELINE - FIXATION subtraction were obtained using data from all three experiments. These were divided into two sets, a hypothesis-generating data set $(n=17)$ and a hypothesis-testing data set $(n=18)$. Again, all peaks of bloodflow change greater than 30 PET counts in magnitude were localized within the summed image of the hypothesis-generating data set (17 increases and 13 decreases). Regions of interest were defined around each of these locations, as described earlier. Each region was then examined using the second, independent hypothesis-testing data set. Mean magnitudes of blood-flow change and variance were calculated for each region. Blood flow in 15 of the regions of increase and all of the regions of decrease produced a significant (or trend, $p<0.1$ ) change from zero (Tables 3,4$)$. The two data sets were then combined into one large-sample image $(N=35)$ to obtain the best estimates of peak locations of blood-flow change (Tables 3, 4; Fig. 7).

Blood-flow increases were observed in extrastriate visual cortex, left-lateralized Rolandic cortex, cerebellar cortex, and supplementary motor cortcx. In addition, a robust blood-flow increase was observed in a region in left lateral prefrontal cortex at or near Brodmann's area 45. Blood-flow decreases were observed in right Sylvian-insular cortex and in parietal cortex both medially and bilaterally.

Regions active in specific experiments. Having identified regions of blood-flow change that were consistent across all three experiments, analyses were next carried out to isolate blood-flow changes specific to each experiment. Summed subtraction im- 


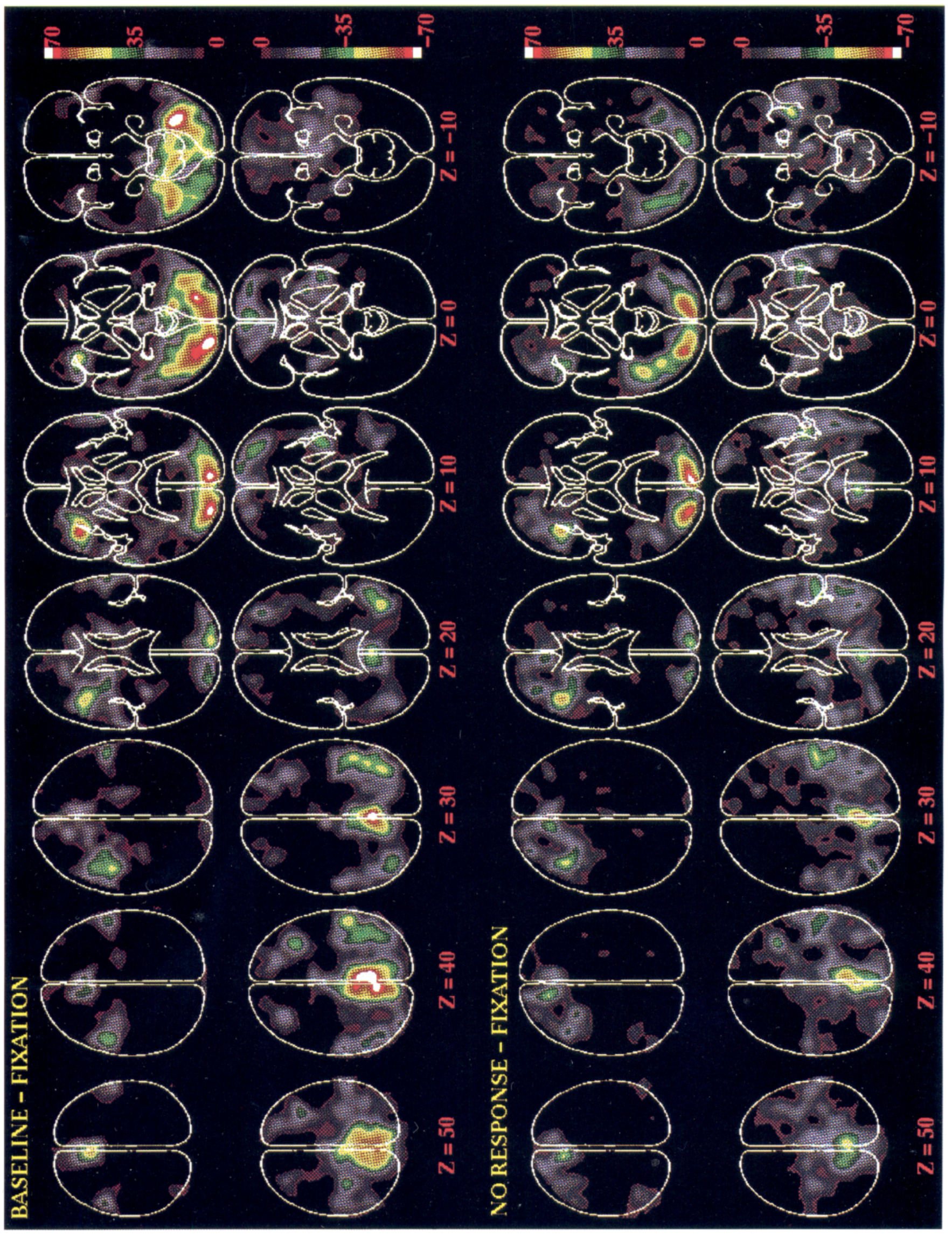


Table 4. BASELINE - FIXATION: hypothesis generation and testing of regions defined around peaks of blood flow decrease

\begin{tabular}{|c|c|c|c|c|c|c|c|c|c|c|c|c|c|}
\hline \multicolumn{4}{|c|}{$\begin{array}{l}\text { Hypothesis generating } \\
\text { data set } \\
(n=17)\end{array}$} & $\begin{array}{l}\text { Hypothesis } \\
\text { testing } \\
\text { data set } \\
(n=18) \\
\text { Significance }\end{array}$ & \multicolumn{7}{|c|}{$\begin{array}{l}\text { Best estimates of locations using the combined data set } \\
(N=35)\end{array}$} & \multicolumn{2}{|c|}{ Additional hypothesis testing } \\
\hline-70 & -5 & -27 & 38 & $p<0.0001^{*}$ & -5 & -31 & 36 & 5 & -48 & 39 & Med. parietal & $p<0.0001^{*}$ & $p<0.0001^{*}$ \\
\hline-65 & 5 & -41 & 40 & $p<0.0001^{*}$ & 3 & -37 & 40 & -3 & -54 & 43 & Med. parietal & $p<0.0001^{*}$ & $p<0.0001^{*}$ \\
\hline-50 & -55 & -17 & 32 & $p<0.0001^{*}$ & -55 & -17 & 32 & 50 & -33 & 34 & R. lat. parietal & $p<0.0001^{*}$ & $p<0.0001^{*}$ \\
\hline-37 & -35 & 23 & 38 & $p<0.01$ & -35 & 25 & 40 & 32 & 12 & 43 & R. prefrontal & $p<0.05$ & $p<0.0001^{*}$ \\
\hline-37 & -27 & 17 & -4 & Trend & -31 & 17 & -4 & 28 & 3 & -4 & R. putamen & $p<0.0001^{*}$ & $p<0.05$ \\
\hline-36 & 59 & -9 & 36 & Trend & 59 & -13 & 34 & -53 & -29 & 36 & L. lat. parietal & $p<0.0001^{*}$ & $p<0.001$ \\
\hline-36 & 33 & -15 & 48 & $p<0.01$ & 33 & -15 & 50 & -30 & -31 & 51 & L. postcentral sulcus & $p<0.0001^{*}$ & $p<0.05$ \\
\hline-36 & -11 & 13 & -10 & Trend & -7 & 17 & -8 & 6 & 3 & -9 & near BA 25 & $p<0.001^{*}$ & NS \\
\hline
\end{tabular}

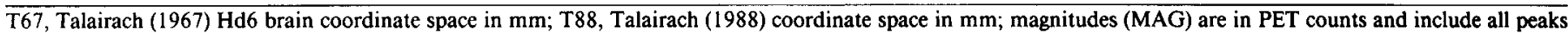

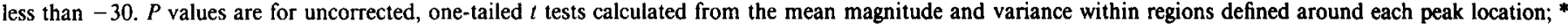

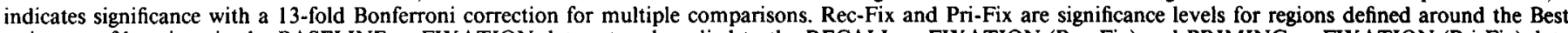

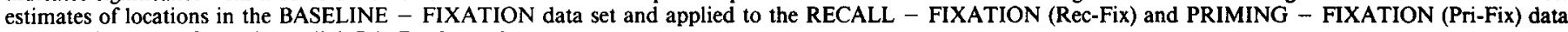
sets. R., right; L., left; Med., medial; BA, Brodmann's area.

ages were obtained for each of the separate experiments (CaseSame, $n=16$; Case-Change, $n=10$; Auditory, $n=9$ ) following the same procedures as in the combined analysis. No additional peak magnitudes of blood-flow change specific to any individual experiment were found that were as large as any of the peaks identified in the combined analysis. However, the signal-tonoise properties of the individual data sets were relatively low compared to the combined data set, especially in the Auditory experiment. Therefore, it is not necessarily the case that there were no differences across experiments.

\section{RECALL - FIXATION subtraction}

The RECALL task was designed to include the processing demands of the BASELINE task and to add processing demands related to explicit memory retrieval. Blood-flow changes related to the memory retrieval demands were isolated in the RECALL - BASELINE subtraction pair, as described above. To test the assumption that the BASELINE task demands were also included in the RECALL task, all of the blood-flow changes identified in the BASELINE - FIXATION subtraction were searched for in the RECALL - FIXATION subtraction $(N=32)$. As previously described, spherical regions were defined around the best estimates of the peak locations of significant or nearly significant (trends) blood-flow changes that were identified in the combined BASELINE - FIXATION image. Mean magnitudes of blood-flow change and variance were then calculated for each of these regions in the RECALL - FIXATION subtraction.

All of the regional blood-flow increases that were significant in the BASELINE - FIXATION subtraction were again significantly increased in the RECALL - FIXATION subtraction
(Tables 3,4). One of the two regions that showed a trend for an increase in the earlier analysis, located in the anterior cingulate gyrus, was significantly increased in the RECALL - FIXATION subtraction. The trend for an increase in blood flow in the medial dorsal thalamus was not present in the RECALL FIXATION subtraction. Similarly, almost all of the blood-flow decreases identified in the BASELINE - FIXATION subtraction were significantly decreased in the RECALL - FIXATION subtraction. The only decrease in the BASELINE task that did not extend to the RECALL task (see Table 4) was located in right anterior prefrontal cortex, near the location found to be increased in the RECALL - BASELINE subtraction. The behavior of blood flow in this region is reported in Frontal regions across tasks, below.

\section{PRIMING - FIXATION subtraction}

In the PRIMING - BASELINE subtraction image, blood-flow reductions were observed in posterior cortex bilaterally. A bloodflow increase was observed in the right hippocampal region in the Case-Same experiment. The small number of blood-flow changes identified in the PRIMING - BASELINE subtraction suggests that regions activated by the PRIMING task were largely the same as those activated by the BASELINE task. As a direct test of this idea, all of the regions found to differ in blood flow in the BASELINE - FIXATION subtraction were also examined in the PRIMING - FIXATION subtraction $(N=$ $26)$. With one exception, all of the regions continued to differ between these two tasks ( $p<0.05$ ) (Tables 3,4$)$.

Finally, the center of mass search algorithm was applied to the PRIMING - FIXATION subtraction. Among the 10 largest

Figure 7. Using the same format as in Figure 2, horizontal blood-flow sections are shown from the BASELINE - FIXATION and NO RESPONSE

- FIXATION summed subtraction images. 
peaks of blood-flow increase and five largest peaks of bloodflow decrease, no peaks of blood-flow change were identified that were not already identified in the analysis of the BASELINE - FIXATION subtraction.

\section{Frontal regions across tasks}

The analyses described above revealed two robust blood-flow increases in prefrontal cortex that were highly reliable across all three experiments (Fig. 8). First, a region in left lateral prefrontal cortex $(\mathrm{T} 67=41,33,10 ; \mathrm{T} 88=-37,20,11)$ (at or near Brodmann's area 45) was increased in the BASELINE task, compared to the FIXATION task, in all 35 subtraction pairs analyzed $(100 \%)$. Second, an anterior right prefrontal region $(\mathrm{T} 67=-35,61,6 ; \mathrm{T} 88=32,50,6)$ (at or near Brodmann's area 10 in frontal-polar cortex) was increased in the RECALL task, compared to the BASELINE task. In this case, the right prefrontal region was increased in 32 of 36 subtraction pairs analyzed $(89 \%)$. To quantify more precisely the behavior of these two regions across tasks, mean blood-flow changes were obtained for both of these regions in the RECALL - FIXATION, PRIMING - FIXATION, and BASELINE - FIXATION subtractions (Fig. 9). As the Auditory experiment did not contain a PRIMING - FIXATION subtraction, the betweentask regional values werc from partially ovcrlapping sets of subjects. What emerged from these comparisons was that the left prefrontal region was active in the RECALL, PRIMING, and BASELINE tasks, as compared to FIXATION. The large increase in the right prefrontal region in the RECALL task, as compared to the BASELINE task, was the result of a significant decrease in the BASELINE task and a significant increase in the RECALL task $(p<0.01)$.

\section{Discussion}

Three PET experiments were conducted, all of which contained an explicit memory retrieval task in which subjects actively retrieved recently presented study words by using word stems as cues (RECALL task). In all three experiments, the memory retrieval tasks were administered together with a control task that contained many of the task demands of the memory tasks but did not require retrieval of recently learned words (BASELINE task), as well as a low-level control task in which subjects fixated on a centrally presented cross hair (FIXATION task). Two of the experiments also included an implicit memory task in which subjects could complete word stems to form study words when asked to complete the stems to form the first word that came to mind (PRIMING task). Across the three experiments, the relationship between the format of the study words and the memory test cues was varied. Data from the first of the three experiments have been discussed in a previous report (Squire et al., 1992).

\section{BASELINE task}

A large number of brain regions were found to be active during the BASELINE task, compared to the FIXATION control task (Table 3). These regions were active in all three experiments. Consistent with other PET studies (Frith et al., 1991; Wise et al., 1991; Fiez and Petersen, 1993; Petersen and Fiez, 1993; Raichle et al., 1994) and other neurobiological studies, extrastriate cortical regions, Rolandic cortex, medial cerebellar and supplementary motor cortices were all active in the BASELINE task, which required processing visual information and producing a verbal output. Blood-flow increases within extrastriate cortex were bilateral and extended into more anterior regions on the left side. The left-lateralized extrastriate region appeared to include a region that has been observed to be active when subjects view words or pseudowords (e.g., FLOOP), but not when subjects view random letter strings (e.g., DRTKL) or false fonts (Petersen et al., 1989, 1990). These results were interpreted previously to suggest that this region is part of a "system that makes distinctions based on information about the combinations of letters that English words can regularly assume" (Petersen and Fiez, 1993, p 519). In our BASELINE task, the visually presented three-letter strings may activate this region because the letter strings are readily made into English words, and the task requires the subjects to do so. As most of the anterior extent of the left extrastriate blood-flow increase observed in the present study is lateral to this medial extrastriate area (Petersen et al., 1990), it is probable that this blood-flow increase also involves a set of regions that are functionally distinct (Howard et al., 1992).

A region in left lateral prefrontal cortex was also active during the BASELINE task. This region is part of a larger set of regions found to be active when subjects generate an appropriate verb in response to presented nouns (Petersen et al., 1988; Kaichle et al., 1994) and during other generation tasks (Friston et al., 1991; Frith ct al., 1991; Wisc ct al., 1991). Accordingly, this particular region may be used for processes underlying lexical generation and semantic memory retrieval. However, the role of this region in processes outside of the verbal domain has not been fully explored.

Raichle et al. (1994) (see also Fiez, 1992) further proposed that the set of prefrontal regions active during a verb-generation task may be part of a pathway including left prefrontal cortex, anterior cingulate gyrus, and right lateral cerebellum. This pathway guides nonautomatic verbal response selection during the verb-generation task. When this nonautomatic pathway is activated, an automatic pathway that uses Sylvian insular cortex is suppressed. The BASELINE task may depend on this nonautomatic pathway. Consistent with this idea, in the BASELINE - FIXATION subtraction, an increase in blood flow was observed in the anterior cingulate gyrus and a robust decrease was observed in right Sylvian insular cortex. In addition, a large blood-flow increase was located at $\mathrm{T} 67=-33,-43,-10$; T88 $=30,-60,-11$ on the border between right lateral cerebellar cortex and visual extrastriate cortex about $10 \mathrm{~mm}$ superior to the cerebellar response observed in the generation task (Petersen et al., 1989; Raichle et al., 1994).

The suggestion that a similar pathway may be used during our BASELINE task and during the verb-generation task raises the interesting question of what processes the tasks share in common? Both tasks (i.e., BASELINE task and the verb-generation task of Raichle et al., 1994) require the retrieval of words from semantic memory, the production of verbal responses, and both tasks are guided by cues that do not directly identify the lexical item to be produced.

Compared to the FIXATION task, the BASELINE task produced large decreases in blood flow in medial and lateral parietal regions. These decreases may reflect processing regions used during the FIXATION task and not used during the BASELINE task. That is, although the FIXATION task was intended as a low-level control task, it nevertheless may require distinct processing resources. When the FIXATION task is subtracted away from the BASELINE task, the decreases in parietal cortex may reflect a reduction in certain processing demands specific to the 
FIXATION task. Support for this possibility comes from the observation that similar parietal regions are active in the FIXATION task in comparison to another low-level control task in which subjects simply rest with their eyes closed (S. E. Petersen and M. E. Raichle, unpublished observations).

\section{RECALL task}

The RECALL task differed from the B $\Lambda$ SELINE task by adding an explicit memory retrieval demand. In the RECALL task, subjects could not respond based only on reference to immediately present stimuli and a general knowledge of words and language rules. The RECALL task required that the subjects make conscious and intentional reference to previously presented words.

Subjects look longer on average to produce words during the RECALL task than during the BASELINE task. Separate analysis of reaction times for RECALLED words and NONRECALLED words indicated that the longer mean reaction time in the RECALL task could be accounted for by recall failures (Fig. 1). Presumably, subjects tried to generate a word from the earlier study list. If the memory search was successful, the word was produced relatively quickly. If the subject could not produce a study list word, the memory search was abandoned, and subjects completed the stem cue with an appropriate word that they could generate.

The blood-flow data identified an anterior right prefrontal region, in frontal-polar cortex, that was markedly increased in the RECALL task as compared to the BASELINE task across all three experiments. In addition, the RECALL task, in comparison to the FIXATION task, also activated the regions previously described as being part of a nonautomatic, verbal selection pathway, as observed in the BASELINE - FIXATION subtraction. The right prefrontal region, which was more active in the RECALL task than in the BASELINE task, was less active in the BASELINE task than in the FIXATION task. There are at least two possible interpretations of this blood-flow pattern.

One intriguing possibility is that the RECALL task involves the same nonautomatic, verbal response selection pathway as the BASELINE task, and additionally recruits the right prefrontal region for processes related to retrieval from explicit memory. By this view, the BASELINE task, which did not require subjects to recall recently presented study words, depended on the verbal-response selection pathway, including left prefrontal cortex. Activity in the right prefrontal cortex was suppressed in the BASELINE task. The RECALL task used left prefrontal cortex, like the BASELINE task, and also used the anterior right prefrontal cortex. Involvement of frontal cortex in the RECALL task is consistent with the finding that frontal cortex lesions can impair performance on explicit memory retrieval tasks (Jetter et al., 1986; Janowsky et al., 1989; for discussion, see Moscovitch, 1982; Moscovitch and Wincour, 1992). Recently, several other PET studies of explicit memory retrieval have also demonstrated similar right prefrontal blood flow changes (Haxby et al., 1993; Tulving et al., 1994a).

The nature of the contribution that the right prefrontal activation we observed makes to explicit memory retrieval will need to be addressed in future experiments. Explicit memory retrieval tasks require processes related to searching for target information and the internal monitoring of whether retrieved information is connected to the specific context of the study episode. The right prefrontal cortex activation may be related to any or all of these processing demands.
A second possibility for the involvement of the right prefrontal cortex in the RECALL task is that the task required subjects to alternate between two strategies. Specifically, the subjects had to engage in memory retrieval and then, if the search was unsuccessful, switch strategies and produce any appropriate word. The right prefrontal region may be involved in alternating between strategies rather than in processes related to one particular strategy. This explanation, however, does not account for the blood-flow decrease observed in the BASELINE task, compared to the FIXATION task.

\section{Blood-flow changes in the medial temporal lobe in the RECALL task}

A reproducible increase in blood flow in right medial temporal lobe structures was previously observed in the RECALL task of the Case-Same experiment (Squire et al., 1992). This increase was also observed in the PRIMING task of the Case-Same experiment (Squire et al., 1992), but was significantly smaller in magnitude than in the RECALL task. The region of activation included the hippocampus proper and the surrounding cortex. We were surprised that a similar increase in blood flow was not observed in the medial temporal lobe in the RECALL task of either the Case-Change or Auditory experiments.

The increase in blood flow in the right hippocampal region in the RECALL task of the Case-Same experiment was originally interpreted to result from the general explicit memory demands required by the RECALL task. However, the results of our CaseChange and Auditory experiments, both of which require explicit memory retrieval, argue against this interpretation. Several other recent PET studies that have examined explicit memory have also failed to detect significant blood-flow changes in the hippocampal region during either encoding (Kapur et al., 1994; Shallice et al., 1994) or retrieval (Haxby et al., 1993; Shallice et al., 1994; Tulving et al., 1994a). Several additional PET studies that have used tasks that are known to produce memory encoding have not reported significant hippocampal region activation (Petersen et al., 1988, 1989; Frith et al., 1991; Wise et al., 1991; for review, see Buckner and Tulving, 1994). These studies employed analysis procedures that surveyed regional blood-flow change across large areas of the brain that, in each case, included the medial temporal lobe.

Jones-Gottman et al. (1993) recently demonstrated right hippocampal blood-flow increases during an olfactory stimulation task that included an explicit recognition component, and Grasby et al. (1993) showed right parahippocampal activation during two tasks, only one of which contained demands on long-term verbal retrieval. Three additional PET studies employing tasks without obvious explicit memory components have also reported right medial temporal lobe activation (Corbetta et al., 1991; Sergent et al., 1992, 1993).

Thus, there is at the moment uncertainty over whether the hippocampal region (hippocampus proper and surrounding cortex) becomes acutely active, as measured by PET, during the performance of explicit memory tasks. Furthermore, it is uncertain whether blood-flow changes in this region, when present, are due to demands related to explicit memory.

This leaves open the question of why the right hippocampal region was active in the Case-Same experiment of our present study. The task requirements were strikingly similar across the three experiments. In all three experiments, subjects were asked to recall recently studied words when presented with uppercase three-letter word stems as cues. It is possible that the findings 

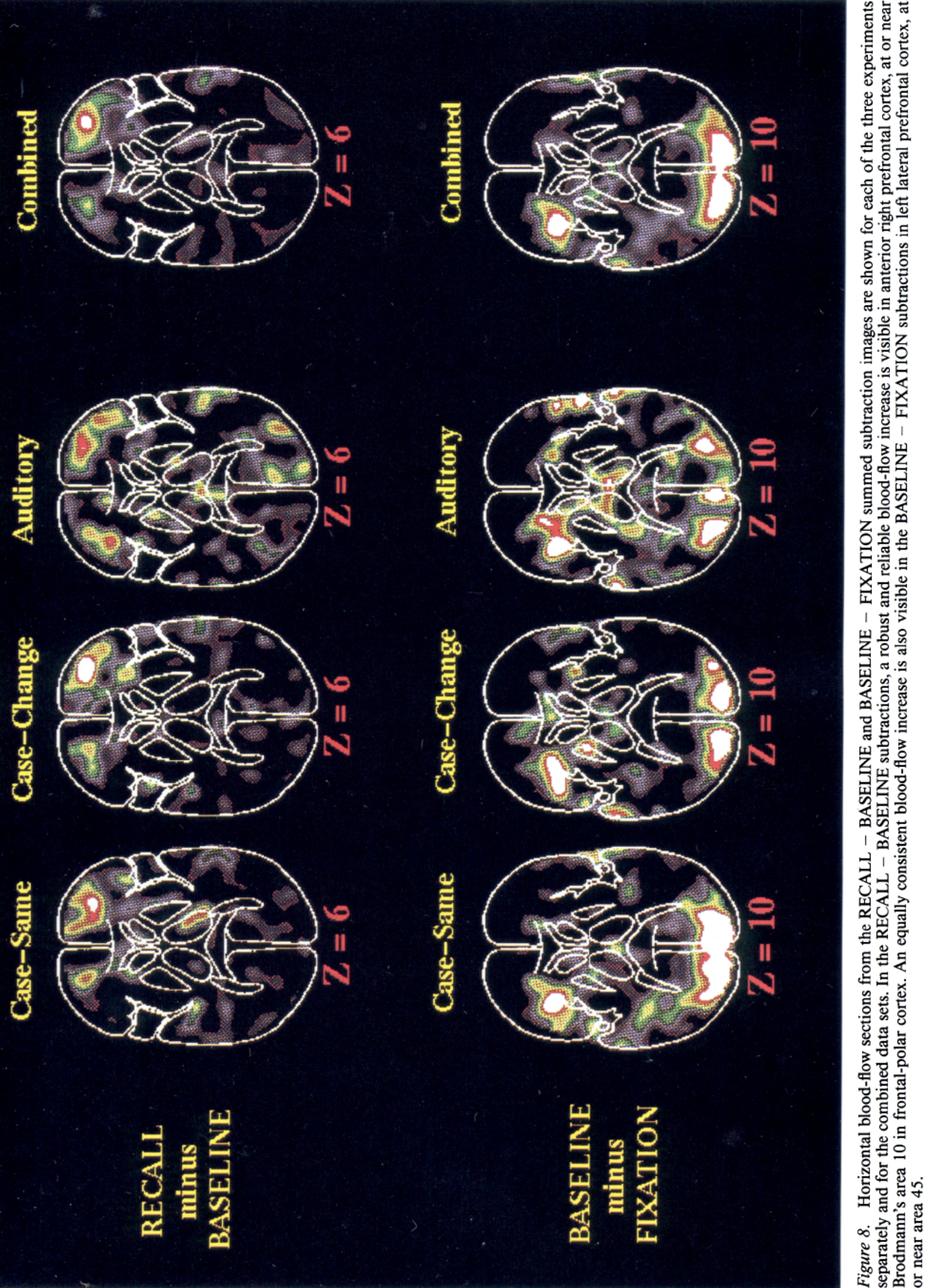
may reflect an interaction between explicit memory processes and other cognitive processes that were specific to the CaseSame experiment. Alternatively, the explanation may lie in cognitive processes other than explicit memory, which were differentially engaged in the three experiments.

\section{PRIMING task}

Unlike the RECALL task, which activated brain areas that were not active in the BASELINE task, the functional anatomy of the PRIMING task and the BASELINE task was nearly identical (Figs. 4, 7). During the PRIMING task, however, subjects tended to complete stems with study words (PRIMED). Voice reaction time was faster for these PRIMED words, compared to the time required to form novel words (NOVEL) in the PRIMING task, and also faster than the time needed to produce words in the BASELINE task. Thus, the PRIMING task resulted in faster performance than the BASELINE task, and there were few qualitative changes in the underlying anatomy.

Nevertheless, there were quantitative changes in blood-flow patterns in the PRIMING task, compared to the BASELINE task. Modest blood-flow reductions were observed in visual processing regions of occipitotemporal cortex, with the rightlateralized reductions being greater than the left-lateralized reductions in both the Case-Same and Case-Change experiments. These behavioral and PET data provide a clue to the neural basis of priming: following exposure to a stimulus, subsequent perceptual processing is more efficient, producing quicker response times and requiring less neural activity. However, the precise nature of the neural changes that underlie this priming effect is not specified by the present data. It is possible that the reductions in the amount of neural activity required to do the PRIMING task reflect the relative ease with which stimuli are processed during the PRIMING task as compared to the BASELINE task and are incidental to the neural changes that have occurred to produce the priming effect.

It was perhaps surprising that the lateralization of blood-flow reductions was the same for both the Case-Same and CaseChange experiments. Marsolek et al. (1992), using lateralized presentation of word stems, found that priming was greater when word stems were presented in the left visual field (right hemisphere) and were in the same lettercase and the same sensory modality as study words. This priming benefit, which they termed "form-specific" priming, was diminished when stems were not visually identical to study words or when word stems were presented in the right visual field (left hemisphere). These behavioral findings are consistent with the right-lateralized bloodflow reduction observed in the Case-Same experiment and with the interpretation that the right-lateralized blood-flow reduction is related to form-specific priming. However, in the Case-Change experiment, a similar right-lateralized reduction in blood flow was observed when form-specific priming could not have occurred. In addition, both the Case-Same and Case-Change experiments showed smaller blood-flow reductions in left-lateralized posterior cortex. It is therefore difficult to draw conclusions about the contribution these posterior regions make to formspecific and more abstract mechanisms of priming.

\section{General conclusions}

The present findings lead to three important conclusions concerning brain activation during memory retrieval.

First, many brain areas, including left lateral prefrontal cortex, right anterior frontal-polar cortex, and anterior cingulate gyrus,

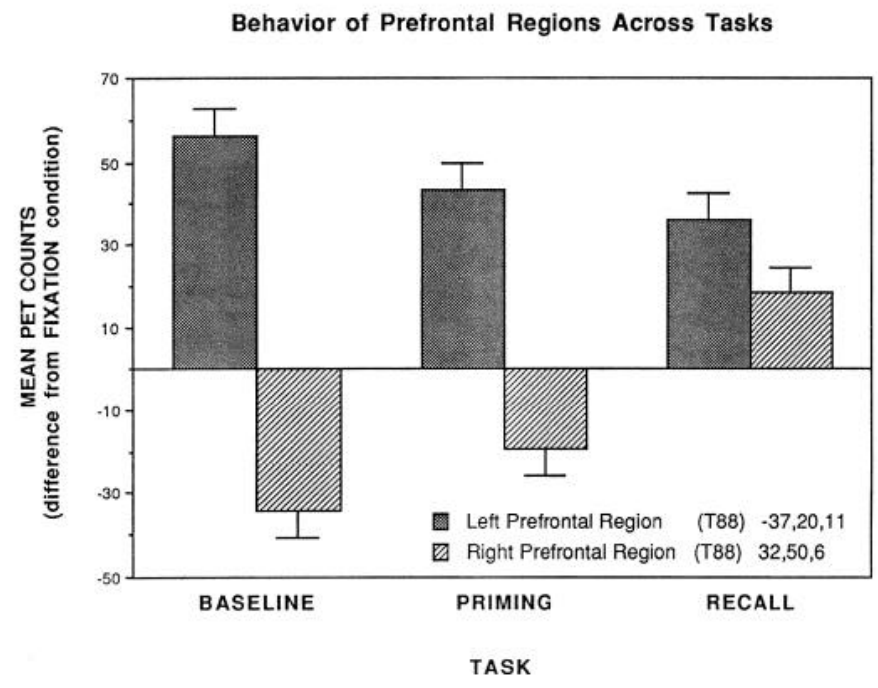

Figure 9. Mean regional blood-flow changes within the right anterior and left lateral prefrontal regions are shown for the BASELINE - FIXATION, PRIMING - FIXATION, and RECALL - FIXATION subtractions. Compared to the FIXATION task, blood flow in the left prefrontal region is increased for all of the three tasks. In contrast, blood flow in the right prefrontal region is decreased in the BASELINE and PRIMING tasks, and increased in the RECALL task.

were active during the explicit memory RECALL task. Except for the right prefrontal areas, these areas were also active during the BASELINE stem completion task and similar areas have, in previous studies, been activated during lexical verb-generation tasks. The finding that a similar set of regions are activated during the BASELINE, RECALL, and verb-generation tasks suggests that a common pathway is recruited during all three of the tasks - possibly due to their shared demands for speech production and semantic memory retrieval. At the same time, the additional areas recruited by the RECALL task suggest that this pathway can be used with other brain areas to accomplish recent verbal memory retrieval. The RECALL task, which required that subjects retrieve words from explicit memory, may have used right prefrontal cortex to aid in processes related to memory search and planning, or for processes related to the internal monitoring of whether a retrieved word was the appropriate target word.

Second, the lack of detectable blood-flow change in the hippocampal region was surprising. The present PET data are consistent with the idea that the essential contribution of the human hippocampal region to explicit memory does not always include an acute increase in activity at the time of retrieval.

Finally, the data identify a neural correlate of priming. Perceptual processing of a stimulus is more efficient after exposure to that stimulus, producing quicker response times and requiring less neural activity.

\section{References}

Buckner RL, Tulving ET (in press) Neuroimaging studies of memory: theory and recent PET results. In: Handbook of neuropsychology, Vol 10 (Boller F, Grafman J, eds). Amsterdam: Elsevier.

Corbetta M, Miezin FM, Dobmeyer S, Shulman GL, Petersen SE (1991) Selective and divided attention during visual discrimination of shape, color, and speed: functional anatomy by positron emission tomography. J Neurosci 11:2383-2402.

Fiez JA (1992) Functional anatomy of lexical processing: PET activation and performance studies. PhD thesis, Washington University. 
Fiez JA, Petersen SE (1993) PET as part of an interdisciplinary approach to understanding processes involved in reading. Psychol Sci 4:287-293.

Fox PT, Mintun MA (1989) Noninvasive functional brain mapping by change-distribution analysis of averaged PET images of $\mathrm{H}_{2}{ }^{15} \mathrm{O}$. J Nucl Med 30:141-149.

Fox PT, Raichlc ME (1986) Focal physiological uncoupling of cerebral blood flow and oxidative metabolism during somatosensory stimulation in human subjects. Proc Natl Acad Sci USA 83:1140-1144.

Fox PT, Mintun MA, Raichle ME, Herscovitch P (1984) A noninvasive approach to quantitative functional brain mapping with $\mathrm{H}_{2}{ }^{15} \mathrm{O}$ and positron emission tomography. J Cereb Blood Flow Metab 4:329333.

Fox PT, Perlmutter JS, Raichle ME (1985) A stereotactic method of anatomical localization for positron emission tomography. J Comput Assist Tomogr 9:141-153.

Fox PT, Miezin FM, Allman JM, Van Essen DC, Raichle ME (1987) Retinotopic organization of human visual cortex mapped with positron emission tomography. J Neurosci 7:913-922.

Fox PT, Mintun MA, Reiman EM, Raichle ME (1988) Enhanced detcction of focal brain responses using intersubject averaging and change-distribution analysis of subtracted PET images. J Cereb Blood Flow Metab 8:642-653.

Friston KJ, Frith CD, Liddle PF, Frackowiak RSJ (1991) Investigating a network model of word generation with positron emission tomography. Proc R Soc Lond [Biol] 244:101-106.

Frith CD, Friston K, Liddle PF, Frackowiak RSJ (1991) Willed action and the prefrontal cortex in man: a study with PET. Proc R Soc Lond [Biol] 244:241-246.

Gabrieli JDE, Milberg W, Keane MM, Corkin S (1990) Intact priming of patterns despite impaired memory. Neuropsychologia 28:417-427.

Graf P, Mandler G, Haden P (1982) Simulating amnesic symptoms in normal subjects. Science 218:1243-1244.

Graf $P$, Squire LR, Mandler $G$ (1984) The information that amnesic paticnts do not forget. J Exp Psychol [Hum Lcarn] 10:164-178.

Grasby PM, Frith CD, Friston KJ, Bench C, Frackowiak RSJ, Dolan RJ (1993) Functional mapping of brain areas implicated in auditory-verbal memory function. Brain 116:1-20.

Haxby JV, Horwitz B, Maisog JM, Ungerleider LG, Mishkin M, Schapiro MB, Rapoprt SI, Grady CL (1993) Frontal and temporal participation in long-term recognition memory for faces: A PET-rCBF activation study. J Cereb Blood Flow Metab 13[Suppl 1]:S499.

Herscovitch P, Markham J, Raichle ME (1983) Brain blood flow measured with intravenous $\mathrm{H}_{2}{ }^{15} \mathrm{O}$. I. Theory and error analysis. $\mathrm{J}$ Nucl Med 24:782-789.

Howard D, Patterson K, Wise R, Brown WD, Friston K, Weiller C, Frackowiak R (1992) The cortical localization of the lexicons. Brain 115:1769-1782.

Janowsky JS, Shimamura AP, Kritchevsky M, Squire LR (1989) Cognitive impairment following frontal lobe damage and its relevance to human amnesia. Behav Neurosci 103:548-560.

Jetter W, Poser U, Freeman RB, Markowitsch JH (1986) A verbal long term memory deficit in frontal lobe damaged patients. Cortex 22:229-242.

Jones-Gotman M, Zatorre RJ, Evans AC, Meyer E (1993) Functional activation of right hippocampus during an olfactory recognition memory task. Soc Neurosci Abstr 19:1002.

Kapur S, Craik FIM, Tulving E, Wilson AA, Houle S, Brown GM (1994) Neuroanatomical correlates of encoding in episodic memory: levels of processing effect. Proc Natl Acad Sci USA 91:2012-2015.

Keane MM, Clarke H, Corkin S (1992) Impaired perceptual priming and intact conceptual priming in a patient with bilateral posterior cerebral lesions. Soc Neurosci Abstr 18:386.

Kucera H, Francis W (1967) Computational analysis of present-day American English. Providence, RI: Brown UP.

Kwong KK, Belliveau JW, Chesler DA, Goldberg IE, Weisskoff RM, Poncelet BP, Kennedy DN, Hoppel BE, Cohen MS, Turner R, Cheng HM, Brady TJ, Rosen BR (1992) Dynamic magnetic resonance imaging of human brain activity during primary sensory stimulation. Proc Natl Acad Sci USA 89:5675-5679.

Lauter JL, Herscovitch P, Formby C, Raichle MR (1985) Tonotopic organization in human auditory cortex revealed by positron emission tomography. Hearing Res 20:199-205.

Marsolek CJ, Kosslyn SM, Squire I.R (1992) Form-specific visual priming in the right cerebral hemisphere. J Exp Psychol [Hum Learn] 18:492-508.
Marsolek CJ, Squire LR, Kosslyn SM, Lulenski ME (in press) Formspecific explicit and implicit memory in the right cerebral hemisphere. Neuropsychology, in press.

Mintun MA, Fox PT, Raichle ME (1989) A highly accurate method of localizing regions of neuronal activation in the human brain with positron emission tomography. J Cereb Blood Flow Metab 9:96-103.

Mishkin M (1982) A memory system in the monkey. Philos Trans $R$ Soc Lond [Biol] 298:85-92.

Moscovitch M (1982) Multiple dissociations of function in amnesia. In: Human memory and amnesia (Cermak L, ed), pp 337-370. Hillsdale, NJ: Erlbaum.

Moscovitch M, Winocur G (1992) Frontal lobes and memory. In: The encyclopedia of learning and memory: neuropsychology (Squire LR, ed). Hillsdale, NJ: Erlbaum.

Ogawa S, Tank DW, Menon R, Ellermann JM, Kim SG, Merkle H, Ugurbil K (1992) Intrinsic signal changes accompanying sensory stimulation: functional brain mapping with magnetic resonance imaging. Proc Natl Acad Sci USA 89:5951-5955.

Petersen SE, Fiez JA (1993) The processing of single words studied with positron emission tomography. Annu Rev Neurosci 16:509-530.

Petersen SE, Fox PT, Posner MI, Mintun M, Raichle ME (1988) Positron emission tomographic studies of the cortical anatomy of singleword processing. Nature 331:585-589.

Petersen SE, Fox PT, Posner MI, Mintun M, Raichle ME (1989) Positron emission tomographic studies of the processing of single words. J Cognit Neurosci 1:153-170.

Petersen SE, Fox PT, Snyder AZ, Raichle ME (1990) Activation of extrastriate and frontal cortical areas by visual words and word-like stimuli. Science 249:1041-1044.

Raczkowski D, Kalat JW, Nebes R (1974) Reliability and validity of some handedness questionnaire items. Neuropsychology $6: 43-47$.

Raichle ME (1987) Circulatory and metabolic correlates of brain function in normal humans. In: Handbook of physiology, The nervous system, Vol V, Higher functions of the brain, Pt 2 (Mountcastle VB, Plum F, eds), pp 643-674. Bethesda, MD: American Physiological Society.

Raichle ME, Fiez JA, Videen TO, Petersen SE (1992) Activation of left posterior temporal cortex in a verbal response selection task is rate dependent. Soc Neurosci Abstr 18:933.

Raichle ME, Fiez JA, Videen TO, MacLeod AK, Pardo JV, Fox PT, Petersen SE (1994) Practice-related changes in human brain functional anatomy during non-motor learning. Cereb Cortex 4:8-26.

Schacter DL (1987) Implicit memory: history and current status. J Exp Psychol [Hum Learn] 13:501-518.

Schacter DL, Tulving ET (1994) Memory systems 94. Cambridge, MA: MIT Press.

Schacter DL, Chiu C-YP, Ochsner KN (1993) Implicit memory: a selective review. Annu Rev Neurosci 16:159-182.

Scoville WB, Milner B (1957) Loss of recent memory after bilateral hippocampal lesions. J Neurol Neurosurg Psychiatry 20:11-21.

Sergent J, Ohta S, MacDonald B (1992) Functional neuroanatomy of face and object processing. Brain 115:15-36.

Sergent J, MacDonald B, Zuck E (1993) Distinct and asymmetric neural networks underlying access to biographic knowledge from faces and proper names: a PET study. J Cereb Blood Flow Metab 13[Suppl 1]:S505.

Shallice T, Fletcher P, Frith CD, Grasby P, Frackowiak RSJ, Dolan RJ (1994) Brain regions associated with acquisition and retrieval of verbal episodic memory. Nature 368:633-635.

Squire LR (1992) Memory and the hippocampus: a synthesis from findings with rats, monkeys, and humans. Psychol Rev 99:195-231.

Squire LR, Ojemann JG, Miezin FM, Petersen SE, Videen TO, Raichle ME (1992) Activation of the hippocampus in normal humans: a functional anatomical study of memory. Proc Natl Acad Sci USA 89: 1837-1841.

Squire LR, Knowlton B, Musen G (1993) The structure and organization of memory. Annu Rev Psychol 48:453-495.

Talairach J, Tournoux P (1988) Co-planar stereotaxic atlas of the human brain. New York: Thieme.

Talairach J, Szikla G, Tournoux P (1967) Atlas d'Anatomie Stereotaxique du Telencephale. Paris: Masson.

Ter-Pogossian MM, Ficke DC, Hood JT, Yamamoto M, Mullani NA (1982) PETT VI: a positron emission tomograph utilizing cesium fluoride scintillation detectors. J Comput Assist Tomogr 6:125-133.

Tulving E (1985) How many memory systems are there? Am Psychol 40:385-398. 
Tulving E, Schacter DL (1990) Priming and human memory systems. Science 247:301-306.

Tulving E, Kapur S, Markowitsch HJ, Craik FIM, Habib R, Houle S (1994a) Neuroanatomical correlates of retrieval in episodic memory: auditory sentence recognition. Proc Natl Acad Sci USA 91:20122015.

Tulving E, Kapur S, Craik FIM, Moscovitch M, Houle S (1994b) Hemispheric encoding/retrieval asymmetry in episodic memory: positron emission tomography findings. Proc Natl Acad Sci USA 91: 2016-2020.

Victor M, Agamanolis J (1990) Amnesia due to lesions confined to the hippocampus: a clinical-pathological study. J Cognit Neurosci 2:246-257.

Warrington EK, Weiskrantz L (1974) The effect of prior learning on subsequent retention in amnesic patients. Neuropsychology 12:419428.
Weiskrantz L (1990) Problems of learning and memory: one or multiple memory systems? Philos Trans R Soc Lond [Biol] 329:99-108.

Wise R, Chollet F, Hadar U, Friston K, Hoffner E, Frackowiak R (1991) Distribution of cortical neural networks involved in word comprehension and word retrieval. Brain 114:1803-1817.

Yamamoto M, Ficke DC, Ter-Pogossian M (1982) Performance study of PETT VI, a positron computed tomograph with 288 cesium fluoride detectors. IEEE Trans Nucl Sci NS-29.

Zola-Morgan S, Squire LR (1993) Neuroanatomy of memory. Annu Rev Neurosci 16:547-563.

Zola-Morgan S, Squire LR, Amaral DG (1986) Human amnesia and the medial temporal region: enduring memory impairment following a bilateral lesion limited to field CAl of the hippocampus. J Neurosci 6:2950-2967. 